This manuscript is a preprint and has not been submitted for publication.

A previous of the work version appears in the first author's doctoral dissertation (http://doi.org/11122/10542) and includes several corrections and clarifications to Chapter 4 of that thesis. No plan has been made to submit this manuscript for peer-review, but a standalone preprint version desired in the meantime. The first author welcomes correspondence regarding questions, comments, criticism, concerns, and other feedback at jnstroh@alaska.edu. 


\title{
Variational Ensemble-Transform Filtering of HFR \& ADCP velocities in the ice-free Chukchi Sea
}

\author{
J.N.Stroh, Gleb Panteleev, and Tom Weingartner
}

2019-04-19

\begin{abstract}
The Chukchi Sea (CS) is the gateway to the Arctic Ocean (AO) for Pacific waters entering from Bering Strait (BS) and also a potential location for future offshore oil extraction. Since 2010, regional CS data has become more plentiful with acoustic Doppler current profilers (ADCP) moored throughout the northwestern portion of the shelf along with coastal high-frequency radar (HFR) surface current monitoring during the ice-free summer season. This work develops a data assimilation system (DAS) for these observations which applies an asynchronous variational ensemble filter to a Regional Ocean Modeling System (ROMS) CS domain. Two configurations of the DAS applied during August-November 2012 are tested and compared with observations from several sources, including unassimilated external data. The tested DAS configurations performed when assimilating surface as a full timeseries of observations rather than as forecast-interval means. The resulting system could be used for future operational forecast refinement in the region well suited for application to surface monitoring and forecast for regional oil spill mitigation. Failures of background model which limit further analysis are discussed in appended material.
\end{abstract}

\section{Introduction}

The Chukchi Sea (CS) is an essential constituent of the Arctic Ocean (AO) where Pacific waters entering through Bering Strait (BS) conflow with water masses originating from the Atlantic Ocean and the Siberian Shelf, the Canada Basin, and seasonal sea-ice. In addition to its key role in the $\mathrm{AO}$ freshwater and heat budgets, the region is also important to 
resident and migratory wildlife, potentially subject to energy development, and likely to see increased commercial maritime activity in the coming years. At present, the region lacks an operational surface monitoring forecast system suitable to aid in mitigation of oil spills or other advected contaminants. Such considerations motivate attentive monitoring of the region and the development of possible data-informed forecast systems.

The CS is shallow, with depth rarely exceeding $60 \mathrm{~m}$, but lies above a broad continental shelf with area roughly $770^{2} \mathrm{~km}^{2}$ and contributes over half the total coastal water territory of the USA. Regional flow is primarily by the sea-surface geopotential difference between the North Pacific and Arctic Oceans (Coachman et al., 1975; Woodgate et al., 2005) which is strongly regulated by both large-scale atmospheric dynamics (Danielson et al., 2014; Peralta-Ferriz and Woodgate, 2017). Circulation through the CS is governed by topographic depressions which trifurcate the incoming BS northward flow into three channels: a western flow through Herald Canyon (Pickart et al., 2010; Itoh et al., 2012; Gong and Pickart, 2015), a flow through the Central Channel (Weingartner et al., 2005), and the Alaska Coastal Current (ACC). A local map of the region and flow may be found in Weingartner et al. (2005). Relative distribution of flow through each branch varies with seasonal changes in wind forcing and strength of baroclinic flow components. Higher frequency flow modulation results from local wind forcing (Weingartner et al., 1998, 2017a), external inflow variation (Woodgate et al., 2005; Danielson et al., 2014), and baroclinic effects from the presence of different watermasses (Pisareva et al., 2015; Pickart et al., 2016).

In the eastern CS, Hanna Shoal together with minor topographic features and the continental shelf break influence the CC to merge with the ACC near the northernmost reach of the Alaska coast. This common flows reaches Barrow Canyon (BC), a nearshore along-coastal depression that serves as the major entrypoint for relatively warm Pacific and post-Eurasian flow Atlantic waters to the Arctic basins. Itoh et al. (2013) estimates annual flow through Barrow Canyon as $0.45 \mathrm{~Sv}$ near the mouth with much higher rates of transport ( 1.0Sv) in summer when winds are coherent with the stronger background pressure gradient than in winter $(\sim 0.1 \mathrm{~Sv})$ when southward-blowing winds oppose a weaker pressure gradient. Okko- 
nen et al. (2009) found that flow into Barrow Canyon is strongly modulated by wind and buoyancy effects of the source ACC flow. Williams et al. (2014) investigate water-mass exchanges over the shelf-breaks along the boundaries of the CS, while more recent work by Corlett and Pickart (2017) studies the current structure along the shelfbreak. Many of these studies have been aided by moored acoustic Doppler current profilers (ADCP, or moorings) and coastally-installed high-frequency radar (HFR) to monitor circulation over the region.

The earliest data assimilation (DA) study in the Chukchi region which combined observational data and numerical modeling into a DA system (DAS) reconstructed the ecohydrology of the north Bering and southern Chukchi Seas using the 3-dimensional variational (3DVar) assimilation method (Brasseur and Haus, 1991). More recent regional DAS applications focus on: optimal north Pacific state reconstruction (Awaji et al., 2003), circulation of the Bering Sea and model sensitivity to moorings (Panteleev et al., 2009), Chukchi circulation during data-rich years 1990-1991 (Panteleev et al., 2010), reconstruction of Bering Sea SSH (Panteleev et al., 2011) and circulation (Panteleev et al., 2012), configuration and optimization of HFR sites for Bering Strait monitoring (Panteleev et al., 2013, 2015), and CS thermal state regime reconstruction for 1941-2008 (Luchin and Panteleev, 2014). A more recent work by Francis et al. (2017) applies a DAS to examine regional sea-ice loss effects on local circulation. These contemporary studies all implement the 4-dimensional variational (4DVar) data assimilation method (Le Dimet and Talagrand, 1986) as oceanographic studies generally prioritize reconstructive smoothing over operational forecast (Kalnay, 2003; Gustafsson, 2007).

These recent studies, however, have not used new regional HFR data sources in an assimilative study. Ensemble-based DA methods are implemented into operational or real-time monitoring and forecast system more easily than the 4DVar methodology (which requires a separate adjoint model), and provides better scalability with modern parallel computing resources. This work presents a DAS for the Chukchi Sea using the maximum-likelihood ensemble filter (MLEF, Zupanski, 2005)) and the Regional Ocean Modeling System (ROMS, Shchepetkin and McWilliams, 2005) to assimilate surface velocities measured by HFR and 
timeseries of moored observations. The remainder of this study is presented as follows. Section 2 describes the monitoring sources and observational data. Section 3 provides brief details of the MLEF algorithm and a practical extension to assimilate timeseries of observations. Section 4 describes the model setup, tests and validates the DAS, and presents results.

Section 5 summarizes the work and comments on failures of the background model. Dates herein are written in ordinal date format (YYYY-ddd.dd, per ISO 8601) or are referred to by ordinal day prefixed by 'jd' with the year provided in context.

\section{Observational data}

\section{$2.1 \quad$ HFR}

HFR antenna installations along the North Slope of Alaska have existed since 2010, with operational systems since 2012 near communities of Point Lay $\left(69.74^{\circ} \mathrm{N}, 199.99^{\circ} \mathrm{E}\right)$, Wainwright $\left(70.64^{\circ} \mathrm{N}, 199.97^{\circ} \mathrm{E}\right)$, and Utqiagvik/Barrow $\left(71.38^{\circ} \mathrm{N}, 203.52^{\circ} \mathrm{E}\right)$. Another antenna at Simpson $\left(71.06^{\circ} \mathrm{N}, 205.27^{\circ} \mathrm{E}\right)$ became operational in 2013 to resolve surface currents eastward of Barrow. The monitoring system observes velocities during the summer months to a distance approximately $180 \mathrm{~km}$ offshore; Figure 1 identifies the antenna locations and observable CS region within the model. The antennae broadcast frequencies of 4.75-4.8 MHz correspond to bulk surface observations over an effective depth of about $2.5 \mathrm{~m}$ (Stewart and Joy, 1974).

HFR resolution of $2 \mathrm{D}$ velocity fields requires simultaneous observation by independent antenna, so associated gridded datasets contain both temporary gaps exist due to signal intermittence and persistent gaps due to radar geometry. Regional measurements further suffer night-time pollution from ionospheric backscattering (Teague et al., 2001) between 0600 and 1200 UTC (roughly 10pm-4am local time), which reduces the number of observations during that interval by about half. One expects that HFR-conditioned states show some evidence of degraded coherence with these observations at daily $12 \mathrm{Z}$ analyses. In spite of these uncertainties and limitations, HFR remain among the most cost-effective regional observation systems and it is therefore important to maximize the information collected 
from them. The HFR data is available from the Coastal Observing Research and Development Center (http://hfrnet.ucsd.edu), and consists of hourly-averaged velocity records together with associated geometric dilution of precision (GDOP) fields estimating spatial accuracy degradation (Chapman et al., 1997).

\subsection{Moored ADCP}

In the northeast CS, the Hanna Shoal and Barrow Canyon region have been the locus of moored ADCP installations supported by BOEM, NOAA, and local industry. Figure 1 identifies the locations of moorings during 2012-2014 and Table 1 provides further specific details. Acquired mooring data files include 2D timeseries of velocities which are binned at approximately $1 \mathrm{~m}$ intervals from 2-3 $\mathrm{m}$ below the surface to 8-10 $\mathrm{m}$ above the ocean floor. Per source file documentation, hourly profile representatives result from interpolation with $6^{\text {th }}$ order low-pass Butterworth filtering with a 36-hour cutoff threshold.

\subsection{Drifters and CTD}

Dynamical data from 22 drifters released in the central Chukchi region on 2012-225 and 2012-236 serve as external data for comparison to model and DAS counterpart trajectories. Table 2 provides a record of drifter metadata for reference. The drifter observations, obtained from http://research.cfos.uaf.edu/chukchi-beaufort/data_archive. php, comprise hourly or half-hourly drifter velocity and position measured by satellite. Deployment time and locations were assumed to be the first time and position of each record. However, the first record in each timeseries includes a velocity, and are therefore suspected not to correspond directly with physical deployment. A set of CTD observations are also used for quality assessment of vertical temperature and salinity $(\mathrm{T} / \mathrm{S})$ profiles in the model. 


\section{Assimilation Method}

Data assimilation (DA) is a technical framework for combining numerical modeling and observational data, and is an essential component of modern geoscience. In its most direct form, sequential DA methods use empirical data to constrain and adjust primitive equation model evolution (Kalnay, 2003; Jazwinski, 2007). The objective of a DAS is to determine a model state most representative of provided data, given the uncertainties in those data. Among the most commonly employed DA algorithms are the ensemble Kalman Filter (EnKF) (Burgers et al., 1998; Houtekamer and Mitchell, 1998; Evensen, 2003) and variants, whereby a collection of model iterations statistically approximate the classical Kalman Filter (KF) (which is itself a least-squares optimization method (Sorenson, 1970)). The general idea of KF-type methods is to use an ensemble of solutions to empirically construct model (and/or observational) covariances, from which an minimum-variance unbiased estimator (MVUE) of the joint model-data probability distribution (PD) may be calculated algebraically. Variational methods, in contrast, seek to iteratively identify the mode of this PD and may be more robustly applied in cases where the relationship between model states and observations is nonlinear or involved PDs are non-Gaussian. The standard DA notation and nomenclature of Ide et al. (1997) are assumed here for brevity.

In the maximum-likelihood ensemble filter, correction of the forecast state is defined as a linear combination of $N$ ensemble perturbations about the forecast state (Zupanski, 2005) unlike Kalman-type filters where perturbations are centered around the ensemble mean. Specifically, the analysis is given by $x^{a}=x^{f}+\mathbf{P}^{1 / 2} w^{*}$ where $w^{*}$ is an optimal weight vector for columns of $\mathbf{P}^{1 / 2}$, which is a matrix whose columns are scaled ensemble differences from the unperturbed forecast $x^{f}$. The scaling, by $\sqrt{N}$, is such that $\mathbf{P}^{1 / 2} \mathbf{P}^{T / 2}$ is an empirical rank- $N$ approximation to the full model error covariance $\mathbf{P}$ with $\cdot^{T}$ indicating matrix transposition. The analysis $x^{a}$ is identified by minimizing the common variational cost function

$$
\mathbf{J}(x)=\frac{1}{2}\left\|\mathbf{P}^{-1 / 2}\left(x-x^{f}\right)\right\|^{2}+\frac{1}{2}\left\|\mathbf{R}^{-1 / 2}\left(y^{o}-\mathcal{H}(x)\right)\right\|^{2} .
$$

over the $N$-dimensional subspace $\left\{x^{f}+\mathbf{P}^{1 / 2} w\right\}$ parametrized by $w \in \mathbb{R}^{N}$. Here, $\mathbf{R}=$ 
$\mathbf{R}^{1 / 2} \mathbf{R}^{T / 2}$ is the observational error covariance matrix. The Hessian matrix of $\mathbf{J}\left(x^{a}\right)$ identifies the posterior covariance error matrix (Thacker, 1989). From a Bayes' Rule perspective, this optima is the mode of the posterior PD produced when the forecast model PD is updated on the basis of observations (Purser, 1984; Purser and Parrish, 2003; Wikle and Berliner, 2007) with the analysis state corresponding to the maximum a posteriori estimate. The forecast error covariance square-root factor $\mathbf{P}^{1 / 2}$ is updated to reflect this posterior PD by computing the square-root factor of the Hessian term associated with Equation (1) at the analysis (Zupanski, 2005). The columns of this posterior factor define state variations to initiate the next ensemble forecast step via model integration.

The observation operator $\mathcal{H}$ typically defines a mapping between analysis-time model states and observed data. The nature of many EnKF-like DA schemes allow for representing observations at non-analysis times via linear combinations of the observed forecast $\mathcal{H}\left(x^{f}\right)$ and its observed perturbations $\mathcal{H}\left(x^{f}+p_{i}\right)$ at those times. This correspondence is approximate when $\mathcal{H}$ is nonlinear, and may formally require treatment of temporal covariance among the observations (Sakov and Bocquet, 2018). Filtering observations at times different than the present analysis-time is referred to as "asynchronous filtering" although it could also be referred to as a sequential smoothing (Sakov et al., 2010; Sakov and Bocquet, 2018). For simplified notation in diagrams and figures, the so-called innovation vector $d$ quantifies the difference between observation and model counterparts, with $d^{b g}=y^{o}-\mathcal{H}\left(x^{b g}\right)$ and $d^{f}=y^{o}-\mathcal{H}\left(x^{f}\right)$ used for the background and forecast innovations, respectively.

In the application discussed here, asynchronous observation operators corresponding to HFR and ADCP data are quasi-linear operators which output a 6-hour timeseries of hourly velocities. For a state in the target subspace represented by $w$, the associated model observation is the forecast timeseries plus the same linear combination of observed ensemble perturbations. A formal linearization gives $\mathcal{H}(x)=\mathcal{H}\left(x^{f}\right)+\mathbf{P}_{\mathcal{H}}^{1 / 2} w$ where columns of $\mathbf{P}_{\mathcal{H}}^{1 / 2}$ are the observed (via application of $\mathcal{H}$ ) ensemble variations with respect to the observed forecast timeseries; these empirical quantities are easily output and stored during the ensemble forecast step (i.e. model integration). The approach is an alternative for incorporating all 
records of data without shortening the forecast-analysis DA cycle to 1-hour intervals.

The base algorithm identifies the mode of the posterior PD, rather than finding the best linear unbiased estimator under the constraint of minimum variance as in algebraic KF-type filters (Zupanski et al., 2008). This distinction is of primary concern when involved PDs are non-Gaussian (Pires et al., 2010), so that the posterior mode and variance minimizer differ (Talagrand, 2003). The nature of surface currents as measured by HFR (Ashkenazy and Gildor, 2011) or other means (Bracco et al., 2003) are known to be non-Gaussian, and by extension the presumed error structures (Purser and Parrish, 2003) are as well. This motivates the use of the variational approach rather than algebraic method, as the mode would more robustly represent the general disagreement between the model and observations.

Whereas MLEF directly targets a subspace optima of the 3Dvar cost function given in Equation (1), its asynchronous extension approximately solves the 4 Dvar cost function at the analysis time over the ensemble-spanned subspace. The method circumvents the need for an adjoint model to propagate future-time changes in observed errors to initial-time changes in state. The analysis state and covariance structure among the ensemble perturbations stores information as data is assimilated. In contrast with 4 DVar, this ensemble method offers no correction of the entire model trajectory; the analysis step updates only the instantaneous model state rather than its history over the prior forecast interval. It does, however, provide an estimation of analysis uncertainty at no additional cost.

\section{Results and Discussion}

For this study, the ROMS model domain encompasses the region $[58.76 \mathrm{~N}, 83.34]^{\circ} \mathrm{N} \times[168.12$, $229.28]^{\circ} \mathrm{E}$ with grid-scale of approximately $16 \mathrm{~km}$ at the boundaries tapering to approximately $12 \mathrm{~km}$ over the central $1 / 9^{\text {th }}$ of the domain. The domain is artificially large to maintain ongoing ensemble variations, which are suppressed by low-dimensional dynamics of the Bering Strait, and to limit interaction between the open boundaries and the analysis region. Previous experiments with a smaller domain suffered from instabilities due to the formation of a spurious large scale gyres over the deep northeastern that was driven by numerical boundary 
currents. Extant sea-ice over the shelf is thin and rapidly retreats from the continental shelf during the model period of August-October, and is ignored in the ice-free model configuration implemented here. The area of interest resolved at approximately $12 \mathrm{~km}$ is outlined by a dark grey box in Figure 1; this region is used to localize the model analysis and posterior covariance update. Importantly, the domain intends to be kept reasonably coarse for reduced computational time desirable when employing an ensemble of model instances.

Domain bathymetry is sampled from the Alaska Region Digital Elevation Model v2 (Danielson et al., 2015). The vertical grid comprises 15 terrain-following vertical levels with prescribed Mellor-Yamada Level 2.5 closure scheme. Initial data fields are generated by linear interpolation of Hybrid-Coordinate Ocean Model (HYCOM) analysis GLBa0.08 variables (accessible via open-DAP at https://tds.hycom.org/thredds/dodsC/glb_analysis) to the model grid. An identical method and source generated open-boundary values for the duration of model integration. Boundary behaviors were set as radiation/nudging, Flather, and explicit Chapman conditions for full-depth variables, barotropic velocities, and free-surface, respectively.

The 6-hourly ERA-interim fields $(E C M W F, 2012)$ supply ocean surface forcing during simulation. For each year 2012-2014, background models integration begins at jd180 with fixed boundary values, and forcing undergo a 30-day integration with 90-second timesteps to relax dynamical imbalance. Following this adjustment period, initial HYCOM T/S data was re-prescribed and then integrated from jd180 to jd210 with larger timesteps (2.5 minutes) to achieve a fit between the currents and model parameters without disrupting the $\mathrm{T} / \mathrm{S}$ distribution during cold-start adjustment.

The DAS described in Section 3 was initialized with an ensemble of $N=30$ model instances perturbed by random velocity and free-surface variations throughout the ocean domain 24-hours before the first analysis time. The analysis steps occur every 6 hours through the summer periods jd214.00-310.00, which approximately frame the availability of HFR measurements. The observation error covariance factor $\mathbf{R}^{1 / 2}$ is supplied as a diagonal matrix using to estimated standard errors $\sigma_{m}, \sigma_{h}$ modified as follows. Entries corresponding 
to moored velocities are set to a constant value $\sigma_{m}$, while those for HFR are a constant $\sigma_{h}$ multiplied pointwise by its spatial GDOP factor. Temporally averaged HFR GDOP factors for 2012-215-300 are shown in Figure 2 to illustrate the spatial structure of these uncertainties, although the figure suppresses their temporal variability.

In the described experiments, background $\sigma_{m}$ and $\sigma_{h}$ are set to $0.1 \mathrm{~m} / \mathrm{s}$ and $0.33 \mathrm{~m} / \mathrm{s}$, respectively. With this uncertainty model, zonal HFR observation error components are at maximum approximately $0.16 \mathrm{~m} / \mathrm{s}$ nearshore increasing linearly to $0.2 \mathrm{~m} / \mathrm{s}$ at the furthest observable extent, with meridional error components of $0.14 \mathrm{~m} / \mathrm{s}$ where beams are oriented northward growing to $0.5 \mathrm{~m} / \mathrm{s}$ where each antenna beam has the largest azimuth. Early experiments found that this GDOP scaling of prescribed HFR uncertainty yielded smoother posterior ensemble perturbations less prone to model blow-ups. For both ADCP and HFR, prescribed error scales are considerably larger than documented instrumental uncertainties as they subsume errors associated with gridding and pre-processing observations, errors in model representation of true states, and model-space errors incurred by applying $\mathcal{H}$.

The model re-initialization after each analysis requires that barotropic velocity estimates be recalculated for each ensemble member, which depends on the free surface in the terrainfollowing coordinates. Three-dimensional velocity fields as well the free-surface variable compose the state vector $x$ so that it includes all dynamical fields needed for model update. Appendix A discusses several important details of the numerical implementation and filter configuration.

\subsection{Improved Fit to Assimilated Data}

Section 3 discussed how ensemble-transform filters applied asynchronously may be utilized to assimilate timeseries of observations over analysis windows. The MLEF-ROMS DAS could be configured in several ways depending on whether HFR was represented by hourly timeseries of data (i.e. asynchronously) or as 6-hour temporal means ending at the analysis time (i.e. synchronously). To compare the effects of the different HFR observation treatments, DAS application using otherwise identical initialization and configurations was performed. 
Here, Case 1 assimilated HFR as a vectorized 6-hour timeseries while Case 2 assimilated the average record of that timeseries. A model initialized from the same state as the assimilative model run, but which assimilated no data, is used as a background reference to assess the impact of DA. Figures 5 and 6 schematically show the relation of model observations and measurement data in the asynchronous and synchronous cases, respectively. These schemes differs from the classical filtering method in that model observation timeseries (or its mean) cannot be generally constructed from the analysis-time model state without explicit call to the nonlinear model. Alternately, the mean of the timeseries may also be compared to model observations averaged over the forecast window as in Case 2. In both cases, moored ADCP profiles are always treated asynchronously and assimilated as timeseries, with observation-space possibilities represented by ensemble expansion of the forecast and ensemble histories. Note that throughout this discussion, the identification of Case 2 as "synchronous" is imprecise in that Case 2 observations depend directly albeit implicitly on the full history of HFR data during the forecast period. Nevertheless, this term is used to distinguish it from the explicitly asynchronous approach of Case 1.

Figures 7 and 8 show the temporally-smoothed evolution of uncertainty-weighted differences between observations and DAS forecast states relative to those of the background model. The figures show results of Case 1 and Case 2, respectively, presented by comparing case-to-background error ratios. Results presented in this form do not depend on the number of observations which differ between cases; otherwise, one naturally expects that errors in Case 2 be less than those of Case 1 due to smaller vector length. This effect was noted in early experiments conducted to assess the impact of including the free surface $(\zeta)$ as a state vector component: assimilation of only analysis-time HFR data in runs which included $\zeta$ had errors 1-2\% larger than those which did not because of the slightly increased weight given to the background cost term. However, inclusion of $\zeta$ when moorings were assimilated appeared to reduce the scale of artificial gravity waves generated by analysis changes of the velocity field, yielding smoother forecasts and more stable integration of perturbed models ${ }^{1}$.

\footnotetext{
${ }^{1}$ In the official ROMS modeling forums, the one of the main numerical developers discouraged freesurface data assimilation as it results in a "volumetric buoyancy forcing" which is "non-physical, and you do
} 
Alternate methods of suppressing adjustment waves from the analysis (Barth et al., 2007) were attempted without success.

The results shown in these figures are qualitatively comparable; errors in the background model are reduced by $20-35 \%$ on average. Both DAS applications successfully constrain and correct model trajectories by comparable amounts when considering all forecast-minusobservations (black lines), although the asynchronous case has a clear advantage most of the time. Specifically, in relation to the background errors, Case 1 errors against HFR (ADCP) decrease by $11 \%(32 \%)$ in the mean while the corresponding error(s) for Case 2 reduce by $5 \%(20 \%)$.

The qualitative similarity is expected, as the two representations of HFR data are related directly. However, the corresponding volume of HFR observations is not identical; pointwise HFR observations in Case 1 are about 5 times more numerous than in Case 2. This results in significantly different filter response: the error reduction in Case 1 is balanced between HFR and ADCP errors, while Case 2 total errors closely track the errors in the more numerous ADCP data. Thus, the asynchronous assimilation of HFR helps to even the relative weight of the two observation types. This effect is most pronounced between jd240 and jd250 when HFR observations are most numerous. During this period, Case 1 errors generally decrease from $\sim 85 \%$ to $\sim 60 \%$ while Case 2 errors are maintained at $\sim 85 \%$ relative to the those of the background model.

\subsubsection{Influence of Wind Regimes}

Of key note is the difference in filter response between cases as it depends on the local wind forcing. The mean relative improvement of ADCP errors is $11 \%$ greater than that of Case 2, which is solely due to the method of HFR velocity assimilation. Previous observationbased studies found that sustained winds exceeding $6 \mathrm{~m} / \mathrm{s}$ blowing southeast $\left(240 \pm 20^{\circ} \mathrm{CCW}\right.$ not expect anything good out of it" [A.Schepetkin, posted to the ROMS forum 2011-12-06 (https://www . myroms . org/forum/viewtopic . php?f=14\&t=2475). However, updating $\zeta$ on the basis of velocities produced improved dynamical balance of analysis barotropic states, resulting fewer waves and model instabilities at model-reinitialization. 
from east) coincided with measured surface flow reversal (Weingartner et al., 2013; Potter et al., 2014), and agree with previous modeling showing barotropic flow reversal when winds critically exceed $\sim 6.4 \mathrm{~m} / \mathrm{s}$ (Winsor and Chapman, 2004). In the background model here, trial-and-error exploration suggested that winds directed toward $225 \pm 60^{\circ}$ (measured counterclockwise from east) with magnitude exceeding $5 \mathrm{~m} / \mathrm{s}$ correlate moderately (57\%) with differences between the mean surface $(2.5-10 \mathrm{~m})$ flow and deeper $(10-30 \mathrm{~m})$ mean flows means in shallow regions of the central CS where depth is between 35 and $50 \mathrm{~m}$.

This wider range and lower critical limit are roughly established parameters which have not been optimized, but are qualitatively similar to cited ranges. With a temporal restriction that they persist for more than 30 hours with gaps less than 12 hours ignored, these events are herein referred to as "opposing" winds and are designated by blue wind vectors in Figures 7 and 8. The associated periods are shown in blue-shaded regions of Figure 9 which compares the relative errors of ADCP fit for the two cases. During these periods, Case 1 strongly reduces errors in both HFR and ADCP while Case 2 errors vary with little net reduction. Averaged over such periods, Case 1 relative mean fit to ADCP improves by $\sim 1.8 \%$ /cycle more than Case 2. In the asynchronous case, the larger volume of HFR data better encourages the analysis toward the observed sheared flow. Meanwhile, Case 2 experiences a unique occurrence in which errors for HFR in Case 2 are lower than the overall error. This suggests that the large near-surface errors during this time are strongly corrected in Case 2 at the expense of quality of fit to local moorings (viz. moorings \#23 and \#24).

However, strong conclusions regarding isolated periods must be cautioned to flow-dependence; states are effectively conditioned on all previously assimilated data and are identical only before the first HFR is assimilated at jd214.25. Also, some persistent differences between HFR and DAS forecast may be a consequence of the ensemble-transform methodology. This is to say that a common linear combination of ensemble vectors may not be able to simultaneously adjust direction of the surface flow measured by HFR and the at-depth velocity profiles measured by ADCP when vertically sheared flow variation is not present among ensemble perturbations. One alternative explanation is that the opposing wind events lead 
to more diverse behavior in the ensemble forecasts, which has the effect of increasing the orthogonality among the ensemble variations; this leads to more efficient optimization as resolution of errors in the column span of $\mathbf{P}^{1 / 2}$ is improved.

Nevertheless, periods do exist in where Case 2 outperforms Case 1. This is clear from the red shaded regions of Figure 9, which identifies "supporting" wind events where atmospheric forcing is aligned with the background flow. Such events are characterized here by the following conditions: having eastward wind components exceeding $4 \mathrm{~m} / \mathrm{s}$ or exceeding 2.5 $\mathrm{m} / \mathrm{s}$ when winds are directed within $\pm 8^{\circ}$ of due east, and a duration than 30 hours with gaps less than 12 hours ignored. Averaged over such periods, Case 2 relative mean fit to ADCP improves by $\sim 0.3 \% /$ cycle more than Case 1 although most of this difference is due to faster degradation of Case 1 fit to ADCP. A clear example is the sustained constraint of model behaviour between jd281-289 where wind stress is aligned with the background flow. During this period, Case 2 shows relative errors of around $60 \%$ while errors are around $72 \%$ in Case 1. Interestingly, the HFR errors directly account for a small fraction $(\sim 20 \%)$ of this difference; strong reduction of errors in fit to ADCP accounts for most of this improvement. This coincides with an onshore wind event, so a likely explanation is that the Case 2 optimum strongly fits the coastal and at-depth ADCP data while the more strongly-weighted HFR reduces the quality of fit to those ADCP in Case 1. This period also marks the start of a large systematic disturbance of the domain generated by a short-duration of rapid inflow from the western boundary along the East Siberian Shelf (not shown). The strong pulse enters the domain as a wave, and follows the Russian coastline to the Bering Strait where it disrupts and reverses the Bering Strait northward transport. Transient consequences are felt throughout the northeast Chukchi shelf until dynamical balance is returned around jd295. No attempt was made to correct or condition the boundary data which are interpolated directly from the HYCOM source to the ROMS domain.

A noteworthy observation regarding DAS behaviour is that zonal components are corrected by HFR more strongly than meridional ones, particularly in shallow regions. Primary reason for this seems to be that onshore, cross-isobath velocities frequently present in the 
HFR observations are strongly resisted by potential vorticity balance in the model which tends to direct flow along isobaths in shallow regions. It is further noted that the HFRimposed constraint in shallow regions is stronger due to the increased number of $\sigma$-coordinate levels used to represent modeled observation counterparts.

The difference in case-wise filter performance under the two wind regimes may be explained by examining the spatial distribution of analysis errors relative to HFR during the events. Figure 10 (11) shows the HFR observations (left panel) and analysis errors for Case 1 (upper right panel) and Case 2 (lower right panel) temporally averaged over all periods of opposing (supporting) wind. During opposing winds, surface currents are generally slow with a maximum onshore HFR component of $\sim 25 \mathrm{~cm} / \mathrm{s}$. During this period, Case 1 has a clear advantage across the observed region, particularly along the coast $(8-20 \mathrm{~cm} / \mathrm{s}$ vs. 10-25 cm/s) and western lobe $(4 \mathrm{~cm} / \mathrm{s}$ vs. $7 \mathrm{~cm} / \mathrm{s})$. Significantly, Case 1 errors are comparatively lower over deeper waters above the head Barrow Canyon $(15 \mathrm{~cm} / \mathrm{s}$ vs. $21 \mathrm{~cm} / \mathrm{s})$ and the southern/eastern side of Hanna Shoal $(3 \mathrm{~cm} / \mathrm{s} v s .8 \mathrm{~cm} / \mathrm{s})$; both regions have HFR observations with westward components. This suggests that Case 1, while having simultaneously lower errors against back-flow aligned ADCP measurements, is better at resolving the surface-sheared flow than Case 2.

During supporting wind events, mean HFR observations show larger magnitude observations, and westward velocities are present in the eastern lobe only following the isobaths southward from east of Hanna Shoal toward Barrow Canyon. Analysis-HFR errors under supporting wind events are generally worse throughout the domain except over the shelfbreak (i.e. beyond the $70 \mathrm{~m}$ isobath) in the northeast extent of the HFR observation. Onshore components of averaged HFR observations near the head of Barrow Canyon are in the range $50-70 \mathrm{~cm} / \mathrm{s}$ with a maximum of $1.2 \mathrm{~m} / \mathrm{s}$. For this region, both cases exhibit errors in the range of 10-25 cm/s. However, Case 2 errors are lower than Case 1 near the the shoals and over the central shelf $(5-8 \mathrm{~cm} / \mathrm{s}$ vs. $8-10 \mathrm{~cm} / \mathrm{s})$ and throughout the western lobe $(6 \mathrm{~cm} / \mathrm{s}$ vs. $7 \mathrm{~cm} / \mathrm{s}$ ). The along-isobath band of increased error following the $40 \mathrm{~m}$ isobath is present in both cases, while the HFR observations are approximately orthogonal to isobaths. This 
strongly supports the notation that the model fails to represent cross-isobath flow as the DAS is consistently unable to resolve these flows.

Without onshore and cross-isobath components resolved among the ensemble variations, the DAS cannot improve fit-to-observations in either case. For the observations near the head of Barrow Canyon, the GDOP of both velocity components is low and the observations are given large weight in producing the analysis. When HFR signals have larger and more regular onshore and cross-isobath velocities that are poorly resolved by the model, Case 1 simply has a larger volume of such data to optimize against. The inability to resolve this data in the ensemble variation leads to a degradation of Case 1 fit to all data; the weight of unresolvable components acts as an additional constraint on the asynchronous cost function and inhibits fit-to-ADCP in this case. In contrast, lower volume of such unfittable data has less net weight in the synchronous cost function, so Case 2 is able the fit to the ADCP instead.

The quality of fit to HFR and ADCP seen in Figures 7-9 during the 2012 season is overall better for the case of asynchronously assimilated HFR. Evidence is also presented that during supporting wind events, Case 1 suffers a loss of fidelity with observations due to abundant unresolved velocity components. However, the frequency and duration of these events during summers 2012-2017, shown in Figure 12, indicates that the asynchronous method would be more advantageous overall. That the 2012 ice-free season has the largest number of identified supporting wind days suggests one should expect a stronger benefit of asynchronous HFR treatment in subsequent years.

\subsection{Comparison to external drifter data}

DAS forecasts in this discussion show improvement in model-observation velocity correspondence. Observational data in the form of Lagrangian drifter position and velocity follow flow patterns and are not easily assimilated using the DAS presented here. Instead, the dynamical data from drifters released in the central Chukchi region on 2012-225.42 and 2012-236.71 serve as external data for validation of forecast velocity. The drifter observational data com- 
prise hourly-averaged velocities and position data. Table 2 lists details for each drifter and Figure 1 plots relevant portions of drifter trajectories in purple. Model drifters are tracked at an effective $0.0 \mathrm{~m}$ depth, while physical drifters in the comparison were deployed with drogues at $1 \mathrm{~m}$ depth. This disparity in representative depth is a consequence of an incorrect assumption by the author based on the presence of surface temperature measurements and lack of documentation in the data files. In fact, many of the physical drifters used $10 \mathrm{~m}$ drogues; they are omitted from this discussion but remain listed in Table 2 .

The first record associated with each physical drifter determines the deployment time and location for the corresponding model trajectory. The simulated counterparts of each drifter are calculated from geographical positions output by the model, which are assessed in two ways to determine model fidelity with observations. First, output position data is used to compute a timeseries of hourly mean distances from the observed drifter position. Second, the difference in simulated mean hourly distance is used to calculated average velocity for correlation comparison to velocities identically calculated from drifter GPS data.

Vector correlation, needed for the latter evaluation, typically measures the common variability of a velocity time-series (Davis, 1985; Kim et al., 2009). However, preliminary assessment using direct vector correlation hourly velocities (or 3-hour velocity timeseries) suggested that these comparisons of deviations provided little insight as they do not account for differences in mean flow direction. Instead, a more useful method of scoring first-order model-observation coherence is through a skill that directly compares model-observation differences, rather than a fit of variability. Considered here is a quantity $r(t)$ that measures the relative size and direction of differences at some time $t$ :

$$
r(t)=1-\frac{\operatorname{mean}\left(\left(\mathbf{w}^{o}-\mathbf{w}^{f}\right)^{2}\right)}{\operatorname{mean}\left(\left(\mathbf{w}^{o}\right)^{2}\right)+\operatorname{mean}\left(\left(\mathbf{w}^{f}\right)^{2}\right)}
$$

where $\mathbf{w}=\left[\begin{array}{llllll}u_{1} & v_{1} & u_{2} & v_{2} & u_{3} & v_{3}\end{array}\right]^{T}$ denotes the $1 \mathrm{D}$-vectorization of a short 3-hour timeseries series of $2 \mathrm{D}$ velocities for the forecast and observations. In a more geometric notation, this skill may be written as 


$$
r(t)=1-\frac{\left\|\mathbf{w}^{o}-\mathbf{w}^{f}\right\|^{2}}{\left\|\mathbf{w}^{o}\right\|^{2}+\left\|\mathbf{w}^{f}\right\|^{2}}=\frac{2\left(\mathbf{w}^{o}\right)^{T} \mathbf{w}^{f}}{\left\|\mathbf{w}^{o}\right\|^{2}+\left\|\mathbf{w}^{f}\right\|^{2}},
$$

from which one may see desirable properties such as: $r= \pm 1$ if and only if $\mathbf{w}^{o}= \pm \mathbf{w}^{f}$, and $r=0$ if and only if $\mathbf{w}^{o} \perp \mathbf{w}^{f}$ and not both zero. The values of $r(t)$ are calculated at 3-hour intervals using 3-hour timeseries of hourly velocities. The quantities are referred to herein as "correlations" as they have properties similar to those of a correlation coefficient, and correspondingly express fractional values as percentages. Nevertheless, this naming is formally incorrect as $r(t)$ measures coherence among magnitude and direction rather than among second-order moments.

Figures 13 and 14 show the mean evolution of distance and correlation metrics for drifters deployed on jd225.42 and jd236.71, respectively, of 2012. The former are deployed in the vicinity of Hanna Shoal while the latter are deployed offshore north of the Alaska Coastal Current ( $c f$. Figure 1). In the region west of Hanna Shoal, simulated drifters in both Case 1 and Case 2 remain closer to the physical data than those of the background model, with Case 1 diverging from the observations at $28 \%$ the rate of the background. The improvement in Case 2 is modest compared to Case 1, as it assimilates less voluminous HFR data in the region. Note the periodic oscillations in the graphed distances, which likely result from inertial oscillations in the data. The DAS forecast oscillations are larger than those of the background, especially in Case 1. Small-scale oscillatory behaviour of the DAS forecasts in the region appears to persists until around jd231 when constraint by moorings \#25 and \#26 begins; conditioning the analysis on these additional data appears to limit the generation of gravity waves and artificial inertial oscillations caused by corrections to surface velocities from assimilated HFR observations. Note that there is a large temporal gap in HFR data during jd220-224.75 ( cf. Figures 7), so both DAS forecasts starting at jd225.25 may still be in the process of adjusting to a relatively large change in model state. With regard to velocity correlation, all simulated solutions rapidly decorrelate in the first 3-hours after deployment reaching to as low as $9 \%$ in Case 1. However, the Case 2 and the background reach zero uncorrelated after 11 hours whereas Case 1 maintains positive correlation until around 19 
hours. At further times after deployment, correlations in all cases oscillate about zero with amplitudes of about $20 \%$.

Figure 14 shows model correspondence with drifters deployed north of the Alaska Coastal Current south of Hanna Shoal. Improvement of DAS solutions over the background model are evident in both plots, with Case 1 again showing advantages over Case 2. Case 1 diverges from the observation $34 \%$ more slowly than the background case over the 2-day period following deployment, and $51 \%$ more slowly over the first 30 hours. In contrast, the $10 \%$ relative divergence rate reduction is a modest $10 \%$. Background, Case 2, and Case 1 solutions remain within $12 \mathrm{~km}$ (corresponding to the width of one local grid cell) of the observation for approximately 22 hours, 28 hours, and 39 hours, respectively. Distance in Case 1 remains less than half that of the background case for the first 42 hours after deployment. With regard to correlation, the $r$-metric for Case 1 decays linearly from $100 \%$ to $50 \%$ over the 27 hour period following deployment. In contrast, the background and Case 2 solution drifters show oscillation in their metrics with periods of approximately 5.5 hours; the mean \pm amplitude for these curves are $41 \pm 11 \%$ and $54 \pm 22 \%$ during the first 24 hours. The corresponding lack of oscillation in distances suggests that the background and Case 2 velocities are out of phase with inertial oscillation present in observations while Case 1 velocities are in phase. DAS correlations are stronger here than for the the jd225.41 drifter group, which is owed in part to the regularity of HFR data; the region is closer to the antenna and thus the DASs are better informed by HFR. And while the behavior of the correlation metrics varies between the DAS cases, their strong qualitative similarity is likely due to identically assimilated data from nearby moorings ( $c f$. Figure 1) combined with a background model that performs moderately well in the region.

The analysis in this section is based on the average of 4-5 drifters deployed en masse, and do not reflect tracking of individual drifters. One notes that the physical drifters are tracked in periods when model forcing contains strong and abrupt changes in wind direction, during which the HFR errors in both cases exhibit large intermittent errors ( $c f$. wind profiles in Figures 7 and 8) which may have diminished the tracking performance of surface drifters. 
Correctly specifying drifter depths should greatly improve the quality of these results, as the comparison here takes place between $1 \mathrm{~m}$ observations and $0 \mathrm{~m}$ simulations. Such improvement would apply in both background model and DAS cases, with stronger improvements expected in the latter where assimilated HFR more appropriately reflects $1 \mathrm{~m}$ velocities (i.e. those actually influencing the drifter) than the $0 \mathrm{~m}$ surface velocities assumed here. Further, the excluded 10m-drogued observations are likely to have less noise and longer-scale spatiotemporal variability, which suggests they may be better represented by coarse model representatives than drifters nearer the surface. Properly tracking depths of the $1 \mathrm{~m}$ and 10 m drifters awaits future DAS runs, as the approximate streamline tracking is not achievable by post-processing.

\section{$5 \quad$ Remarks and Summary}

The work focuses on the development of a DAS for assimilating HFR and ADCP data in the Chukchi Sea. The system consists of an ice-free ROMS model enveloped by a modified ensemble filter. The implemented method is based on MLEF, which variationally identifies the optimal analysis as the maximum a posteriori estimate, modified to assimilate timeseries or synchronous representatives rather than observations directly derived from the analysistime model state. The resulting asynchronous variational ensemble filter is a sequential approximation to the 4DVar method for observations such as HFR surface currents which are known to be non-Gaussian and for which algebraic Kalman-type filters may be ill-suited.

The study compares a pair of DAS results which differed only in treatment of HFR data; Case 1 treated HFR observations over the forecast period asynchronously as a vectorized historical timeseries while Case 2 treated them as a synchronous average observation. Both methods rely on the history of observations and ensemble of observed model counterparts, unlike the traditional filter methods which consider only data at the time of analysis. ADCP data were consistently assimilated as timeseries in both cases. The results were then compared to available 2012 data to assess the quality of improvement, and to diagnose failures. The findings support that the analysis resulting from fully-asynchronous filtering surpasses 
that of the averaged case. Both cases improve upon the forecast quality of the background model and unpresented early cases which assimilated data using the classical (instantaneous) approach, which ignores 5/6 of surface observations when a 6-hour forecast/analysis cycle is used.

Compared to ingested 2012 data, the asynchronous approach to assimilation was shown to have advantages over the averaged approach. In particular, assimilated HFR timeseries yielded a stronger reduction in forecast-minus-observation errors compared to the background model background than averaged HFR. Significantly, asynchronous assimilation of HFR improved the Case 1 analysis fit with ADCP observations by $12 \%$ more than Case 2 relative to the background model errors. The direct comparison of ADCP errors (co-relative to associated errors in the common background model) shows that the assimilation of HFR timeseries has the effect of simultaneously improving overall fit to ADCP observations and HFR observations despite the larger relative weight given to HFR observations in Case 2. On this point, it is noted that increasing model spatial resolution is an alternate method of naturally changing the balance between the number of HFR and ADCP observations; the former increases with lateral model resolution whereas the latter does not.

The scale of overall error improvement is difficult to quantify consistently due to temporal irregularity of regional dynamics and volume of available data, and also because of the flowdependent nature of sequential filtering. However, the magnitude of difference between case-wise DAS improvements is generally greatest during times when local winds oppose the prevailing background flow or contain a strong onshore component ( $c f$. jd240-260 and jd280-290 of Figures 7 and 8). Averaged over these intervals, Case 1 errors for HFR (ADCP) are $14 \%(16 \%)$ lower than those of Case 2.

The quality of fit to observation was shown to vary with wind regime, with Case 1 more advantageous when strong winds induced vertically-sheared flow against the background flow. Strong optimization constraint imposed on the filter by onshore, cross-isobath HFR observations unresolved by the model (and thus the observed ensemble variations) under eastward blowing winds is implicated in the observed degradation of model-observation fidelity for 
Case 1 during such events. However, the spatiotemporal distribution of winds in recent years suggests that local summertime forcing is predominantly shear-inducing, and suggests that the asynchronous treatment of observations is appropriately suited to the region.

Unassimilated Lagrangian drifter observations provided an external reference for comparing the DAS forecasts. For drifters released offshore, the case with fully asynchronous assimilation diverge from observed data $34 \%$ slower than the background model, compared with a more modest $\sim 10 \%$ reduction in rate when ingesting averaged HFR. Case 1 drifter position remained within $12 \mathrm{~km}$ ( 1 grid-width) of observations for an additional 13 hours with positive velocity correlation for an additional 8 hours, while the corresponding improvements in Case 2 were 1 hour and 0 hours, respectively. Results for drifters released nearer to the coast were similar, with the interesting result that Case 1 velocity correlation tended to decay linearly (at a rate of $\sim 2.1 \% / \mathrm{hr}$ ) for the first day rather than oscillating. This trajectory correspondence between DAS forecast and unassimilated drifter data supports the use of asynchronous filtering for ongoing regional application.

An alternate scheme for applying the presented asynchronous ensemble-transform DA involves optimizing the initial model state rather than the forecast state. In this approach, the optimal ensemble-expansion coefficient vector ( $w^{*}$ in Section 3) may be used to define an optimized initial condition for each model integration step, with the analysis state defined by the integrated optimum. The resulting trajectory would have improved fit to the data on which it is conditioned. Additionally, the states generated in this way will be fully modelconstrained as in the strong 4DVar method. This contrasts the presented method in which linear combinations of constrained states are not guaranteed to satisfy nonlinear primitive equations. The initial state in this case would be conditioned on data at future times, and it would more properly be considered a smoother rather than a filter. The implementation would require only minor modifications of the current DAS, although it would double the total model integration time as each model instance is propagated twice between each analysis cycle. Work in this direction is ongoing.

As metrics for the DAS effectiveness in 2012, the work compared DAS forecast quantities 
with those of a background model which assimilates no data. The DAS configuration of Case 1 was found to strongly improve the quality of fit to observations, and it was applied to equally configured ROMS models of 2013 and 2014. Poor background model dynamics were evident, especially with respect to $\mathrm{T} / \mathrm{S}$ distributions and mass/volume transport estimates which clearly do not correspond with observations beyond the analysis subregion; see Appendices B \& C. The employed model inaccurately resolves Bering Strait inflow in terms of both volume transport and vertical freshwater distribution. Crucially, the model omits the Yukon River, which is a significant contributor to both. These background model failures must be corrected when considering a region which hosts a confluence of waters climatically important for the Pacific sector of the AO, and preclude the inclusion of sea ice for extending the modelable season.

The systematic problems with the model affect both the background model and model component of the DAS equally. This justifies the approach taken in this work, which compares different DAS outputs in relation to a common background model. A more properly configured model would obviously produce a more accurate background trajectory. It would would also serve as a better basis for assimilation schemes, such as those explored here, which are primarily developed to constrain and refine model states via temporally-independent corrections rather than overcome persistent model bias (Dee and Da Silva, 1998; Dee, 2005). However, the variational formulation of MLEF permits inherent correction of the bias component in the span of $\mathbf{P}^{1 / 2}$, which implies that a bias-aware version of the algorithm must account for this component. Specifically, the bias-adjustment methods of Dee (2005) are formulated for KF-type methods which define the forecast as the ensemble mean, and adjust that forecast based on a non-zero of mean of the posterior innovation (i.e. mean $\left(d^{a}\right)$ ) computed prior to the forecast step. Further experimentation is necessary to implement such a correction in variational form for MLEF, which requires a different relationship between the forecast state and ensemble perturbations. Bias-aware modification to the DA component cannot, however, correct model deficiencies originating outside of the analysis region, such as the poorly modeled BS flow. 
Ensemble filtering offers a forward-model only method of assimilation which easily scales as computer resources become available, making them more practical than than strongconstraint variational methods for operational forecasting. As HFR surface observations are known to be non-Gaussian, a mode-tracking objective for optimization should thus be be sought. The variational ensemble filter implemented here satisfies both of these requirements, and is tested in its capacity to resolve surface currents in the Chukchi Sea region by assimilating real data in two ways. The quality of coherence between DAS surface forecast and various forms of velocity data presented indicate the strong candidacy of an asynchronous variational ensemble filter for regional application when timeliness of analysis is crucial, such as the monitoring of surface contamination by shipborne heavy fuel oil or other spills.

\section{Acknowledgements}

The author thanks: R. Potter, E. Dobbins, and H. Statscewich at the University of Alaska Institute of Marine Science for their roles in collecting and pre-processing observational data; D. Nechaev at the University of Southern Mississippi Department of Marine Science at NASA Stennis Space Center for partial support in early stages of this work; and K. Ide and B. Hunt at the University of Maryland for their roles in organizing and leading the 2013 Data Assimilation in Geophysics instructional workshop.

\section{A DAS Configuration}

\section{A.1 Ensemble Generation and Size}

To test the effect of ensemble size on analysis quality of assimilated observations, DAS experiments were conducted employing ensembles with 30,60, and 91 perturbed members, respectively. This test configuration assimilated 6-hour mean HFR data and hourly ADCP timeseries, but did not include the free-surface variable $\zeta$ in the model state vector. A base ensemble of 30 perturbed members was produced by adding random noise to the initial 
background state at day 210, integrating for varying number of 3-hour increments to define a variation about the background state at jd214. An additional set of 30 members was generated by adding random noise with a $60 \mathrm{~km}$ decorrelation length scale at jd210 and propagating to jd214. Initial standard deviations of noise added to the velocity fields in these two cases was $10 \mathrm{~cm} / \mathrm{s}$, with the latter smoothed by a 5 -gridpoint radius Gaussian filter to the imposed noise. Finally another 31 perturbations were created by adding random noise (mean amplitude 5\%) to Fast Fourier-transformed copies of the background state at jd214 to generate 31 additional ensemble elements with smooth spatial variations. Figure 3 shows that additional members of the ensemble did not improve the quality of the forecasts in an evident way. One concludes that a 30-member ensemble of forecast variations is sufficient, although some intermittent improvement $(<2 \%$ mean) for HFR is possible at the cost of doubling or tripling total model integration time.

\section{A.2 Approximate Optimization}

Minimization of the nonlinear cost function $\mathbf{J}$ with respect to the control vector $\xi \in \mathbb{R}^{N}$ is at the heart of the analysis. In relation to the variable $w$ discussed in Section 3,

$$
\xi=\left[I_{N}+\mathbf{Z}\left(x^{f}\right)^{T} \mathbf{Z}\left(x^{f}\right)\right]^{1 / 2} w
$$

gives the ensemble-transform coefficient in a Hessian pre-conditioned form $\xi$. This changeof-variables intends to make the control space isotropic by scaling the ensemble expansion coefficients according to their correlation structure. The analysis optimization step of the ROMS-MLEF DAS implements a secant line search algorithm (Wright and Nocedal, 1999) to iteratively update the control variable $\xi$ in sequentially orthogonal subspaces determined by a conjugate gradient (CG) method, closely following Navon and Legler (1987) and Zupanski et al. (2008). To check to effectiveness and efficiency of this approach (identified to as "NLCG-ss") compared to an immediately accessible method, an optimal analysis is found by computing $\arg \min |\nabla \mathbf{J}|$ over the control space using the internal MATLAB function "fminopt". 
The left plot of Figure 4 illustrates the small difference $(<3 \%$ mean over the 90-day period) in the quality of the analyses produced by the search-based and proprietary optimization methods. However, the right plot of the figure demonstrates that mean computation times differ significantly. The NLCG-ss and "fminopt" methods average 78.4 and 486.4 seconds per analysis, respectively. Assimilation cycles at which the optimization times are similar correspond to instances of few observations. The difference in computation time accumulates to nearly 40 hours over the 90-day period shown, roughly doubling the total time needed to apply the 30-element DAS using 20 CPUs.

\section{B Comparison to external CTD data}

While velocity and circulation and their relation to assimilated data are of specific interest, it is worthwhile to consider the quality of other hydro-dynamical circulation aspects within the model. A collection of $\sim 250$ conductivity-temperature-depth (CTD) instrument casts taken during 2012 surveys of the eastern Chukchi Sea provides a dataset of temperature and salinity $(\mathrm{T} / \mathrm{S})$ observations for further testing. All considered observational profiles are contained within the $12 \mathrm{~km}$-resolution model subdomain; spatiotemporal locations of the data, which were acquired internally from University of Alaska Fairbanks Institute of Marine Science, are hidden for brevity. The T/S observations are interpolated to ROMS $\sigma$-coordinates via cubic splines for comparison to background and DAS model representatives. Figure 15 exemplifies CTD-observed temperature and its associated cubic interpolant which poorly-resolves its thermocline; similar problems exist in representation of salinity observations. Some inherent errors are thus expected, particularly in the area of the pycnocline.

Figure 16 plots the CTD T/S observation representatives and relative differences of the background, Case 1, and Case 2 forecast models. Without respect to geolocation, the chronology of CTD observations shows a general trend toward surface cooling and freshening between jd230 and jd270. Cases 1 and 2 show differences from CTD representatives which look very similar to the background model errors.

Unfortunately, the scale and structure of $\mathrm{T} / \mathrm{S}$ errors in the background model dominates 
the errors of Case 1 and Case 2. Within all models, differences from CTD observations increase in time, with profiles progressing toward vertically uniform T/S distributions. Figure 17 shows $\mathrm{T} / \mathrm{S}$ profiles from observations and models 40 days apart to illustrate this problem. Correction of this behavior was attempted by changing vertical mixing/closure options (from the Mellor-Yamada 2.5-layer scheme to K-profile parametrization or generic length-scale mixing) with a variety of different $\mathrm{T} / \mathrm{S}$ mixing options. However, none of these alternatives gave rise to significantly improved vertical $\mathrm{T} / \mathrm{S}$ distribution.

A more in-depth diagnosis is warranted; three appropriate places to begin investigation are the external HYCOM used for initial/boundary data, the vertical coordinate distribution selected in the model, and the evolution of vertical structure at the point of Bering Strait inflow. Cursory topical analysis shows that modeled Bering Strait inflow T/S is unstratified, whereas the HYCOM initialization data resolves a surface freshwater layer several meters thick. The loss of a surface freshwater layer in the model may further reflect the omission of significant freshwater sources, such as the Yukon River discharge averaging $\sim 0.1 \mathrm{~Sv}$ in modeled months per USGS monthly flow rates at Pilot Station, AK. However, this volume is insufficient to balance the model volume BS flow. The strengthening warm bias of modeled temperature profiles compared to CTD is also noted, but its cause is not speculatively diagnosed here in the absence of further experimentation. Such errors and shortcomings of the background model reflect strong systematic biases (Dee and Da Silva, 1998; Chepurin et al., 2005; Dee, 2005), and cannot be corrected by traditional assimilation of $\mathrm{T} / \mathrm{S}$ data which only serve as model constraints. Improvement of the background model to include meteorologic freshwater sources and preserve vertical stratification over the Chukchi Shelf is obviously necessary.

\section{C (Failed) Transport Estimates of Summers 2012-2014}

Figure 18 identifies a set of model transects defined for posterior estimation transport of volume, heat, and freshwater. Each transect is oriented with a northernmost initial point and leftward-normal orientation as the transect is traversed. Each normal direction is thus 
defined with a positive eastward component. Note that the northern Central Channel (CCn) is oriented with the positive side pointing into the region bounded by transects and the coast.

Across each defined transect, vertically-integrated estimates of volume flux $\left(V^{\prime}\right)$, freshwater volume flux $\left(V_{F W}^{\prime}\right)$, and heat flux $\left(Q^{\prime}\right)$ can be calculated from the respective equations:

$$
\begin{aligned}
V^{\prime}(t, l) & =\int_{-h}^{0} u_{\perp} d z \\
V_{F W}^{\prime}(t, l) & =\int_{-h}^{0} \frac{\rho}{\rho_{F W}}\left(1-\frac{S}{S_{r e f}}\right) u_{\perp} d z, \quad \text { and } \\
Q^{\prime}(t, l) & =\int_{-h}^{0} C_{s} \rho\left[\theta-\theta_{r e f}\right] u_{\perp}(x, y, z) d z
\end{aligned}
$$

where $u_{\perp}$ is the velocity component normal to the transect, $\rho_{F W}$ is the density of fresh-water, $C_{s}$ is the state-dependent seawater heat capacity, $\theta$ is potential temperature, and $S_{r e f}$ and $\theta_{\text {ref }}$ are adopted reference values (e.g., $34.8 \mathrm{PSU}$ and $-1.9^{\circ} \mathrm{C}$ are common). Integrals of the fluxes along the length of transect give the associated total transports $V, V_{F W}$, and $Q$.

The gross inaccuracy and unrealistic behaviour of $\mathrm{T} / \mathrm{S}$ in all models disparages their use in calculating $Q^{\prime}$ and $V_{F W}^{\prime}$. Nevertheless, the assimilative model discussed previously demonstrates sufficient coherence with velocity observations and regional dynamics to estimate mass transport. Mass transports are estimated using 24-hour forecast records of velocity data, computed from mean velocities calculated during the DAS forecast step.

\section{C.1 Inflow Sources}

Long Strait (LS) flow is directly related to HYCOM boundary data from HYCOM, with seasonal transport estimates of $5.7 \mathrm{mSv}, 5.5 \mathrm{mSv}$, and $6.9 \mathrm{mSv}$ for the modeled years excluding the anomalous inflow events centered around 2012-223 and 2012-292 outflow event 2014-297. Net eastward transport of those years roughly agrees with estimates calculated using results of Francis et al. (2017).

Regional circulation dependence on Bering Strait (BS) transport is well established (Danielson et al., 2014; Weingartner et al., 2017b). Recent observational studies of moored 
$\mathrm{ADCP}$ find $\mathrm{BS}$ inflow near or above 1.0 Sv in the months of August-October during the modeled years (Woodgate et al., 2015; Woodgate, 2018). However, modeled BS quantities are far lower and typically in the range of $0.45-0.65 \mathrm{~Sv}$. The only months which show near agreement are September and October of 2012, where model (observed) transports are 0.5 $\mathrm{Sv}(0.43 \mathrm{~Sv})$ and $0.41 \mathrm{~Sv}(0.49 \mathrm{~Sv})$, respectfully. (The BS flow reversal around 2012-298 caused by the anomalously strong inflow pulse from the ESS is omitted from October 2012 estimation.) Model results from 2013 are dubious, with vertically-averaged model northward flow through BS in 2013 of approximately $0.25 \mathrm{~m} / \mathrm{s}$ with standard deviations $0.07 \mathrm{~m} / \mathrm{s}$. In other years, the rates generally decreases from $0.6 \mathrm{~m} / \mathrm{s}$ to $0.4 \mathrm{~m} / \mathrm{s}$ over jd214-300 with deviations about that trend of $0.05 \mathrm{~m} / \mathrm{s}$. Current meter estimates from Woodgate et al. (2015); Woodgate (2018) show that realistic flow rates should be roughly twice these values, with model 2013 BS transport underestimated by $\sim 75 \%$. These errors, which are determined by the background model and only slightly influenced by the DAS, could not be corrected by adjusting some model parameters throughout the domain. For example, experiments with the background model showed: decreasing the viscosity from $12 \mathrm{~m}^{2} / \mathrm{s}$ to $1.2 \mathrm{~m}^{2} / \mathrm{s}$ yielded only a $5 \%$ increase in BS flow rate. As previously noted, riverine water sources are ignored but are insufficient to account for the BS flow deficit in the model. The poor transport resolution through BS profoundly impacts the modeled transports throughout the domain.

\section{C.2 Subregional balance estimates}

A consistency check of the model transport estimates shows that the primary CS inflow and outflows are in approximate balance. This is justified by comparing the sum of incoming water from the Long Strait and Bering Strait and the outgoing water across the Barrow Canyon. The seasonal mean differences between these quantities for 2012-2014 are -0.036 $\mathrm{Sv},-0.012 \mathrm{~Sv}$, and -0.023 Sv, respectively. These estimates omit a low-volume source north of Wrangell Island and outflow along shelfbreak current which bypasses Barrow Canyon. An estimate of the latter during 2009-2011 by Brugler et al. (2014) is about 0.02-0.04 Sv, which agrees with the missing component of the budget. 
In 2012 and 2014, flow across the southern Central Channel transect is slightly greater than the concurrent Bering Strait transport of $\sim 0.6 \mathrm{~Sv}$. This suggest that the Siberian Shelf flow volume directed though the southern CCs transect slightly exceeds (by $\sim 0.005 \mathrm{~Sv}$ ) any BS transport flowing northward through Herald Canyon.

The polyline transect composed of the Central Channel (CCn,CCs) transects together with the western transect of the Alaska Coastal Current $(\mathrm{ACCw})$ forms a closed region bounded by the Alaska coast. Forecast transports across the boundaries show an approximate closure, with outflow of through CCs and ACCw accounting for about $97 \%$ of the ACCs inflow across all three years. This error results from a combination of excluded shallow coastal flow, numerical errors in collocating C-grid velocities and bathymetry, and failure to account for changes in free surface. Regional transport distribution in 2012 and 2014 is similar, with northward transport across CCn measuring $16.9 \%$ and $16.8 \%$, respectively, of the incoming flow measured across CCs. The remaining portions, calculated at $80.5 \%$ and $83.1 \%$ respectively, exit the region eastward through $\mathrm{ACCw}$, with standard deviations of about $2 \%$. In 2013, model BS throughout the season is approximately $0.24 \mathrm{~Sv}$ less than the 2012/2014 mean. Consequently CCs inflow is reduced, and the CCn mean outflow is only $12.5 \%$ of the CCs with $82.6 \%$ leaving through $\mathrm{ACCw}$. Local wind forcing does not appear to play a significant role in regulating this balance; correlation coefficients calculated for variations in transport against wind components normal to transects with a 0.5 day lag are uniformly less than $10 \%$.

DAS transport estimates through BC are expected to be inaccurate due to poorly represented BS flow in the background model. Respective 2012-4 seasonal mean flows in the DAS analysis are $1.2 \mathrm{~Sv}, 0.31 \mathrm{~Sv}$, and $0.94 \mathrm{~Sv}$. Ignoring 2013, these contrast with the accurate observational estimates in the $0.45 \mathrm{~Sv}$ range for the head of $\mathrm{BC}$ (Weingartner et al., 2017b) and better align with estimates late-summer flow at the mouth of BC (Itoh et al., 2013). Up-canyon transport events occurs only in 2013, despite the observational expectation of $\sim-0.1 \mathrm{~Sv}$ in the latter half of each modeled season (Weingartner et al., 2017b). Two plausible reasons for this inconsistent behavior involve the model and the DAS itself, 
beyond those induced by BS underestimation. First, the $12 \mathrm{~km}$ model resolution may be insufficient to fully resolve the flow dynamics of the ACC; Okkonen et al. (2009) found that a $9 \mathrm{~km}$ resolution of the ACC was insufficient for simulating the BC regional flow. Second, low BS inflow causes an overall reduction velocities in region where the DAS analysis localized. The data-optimized solution attempts to match data that reflects larger observed velocity components, so that latent bias-adjustment (artificially) increases flow in the ACC and consequently through BC. This latter point underscores the need for BS inflow to be accurately supplied or resolved for regional analysis.

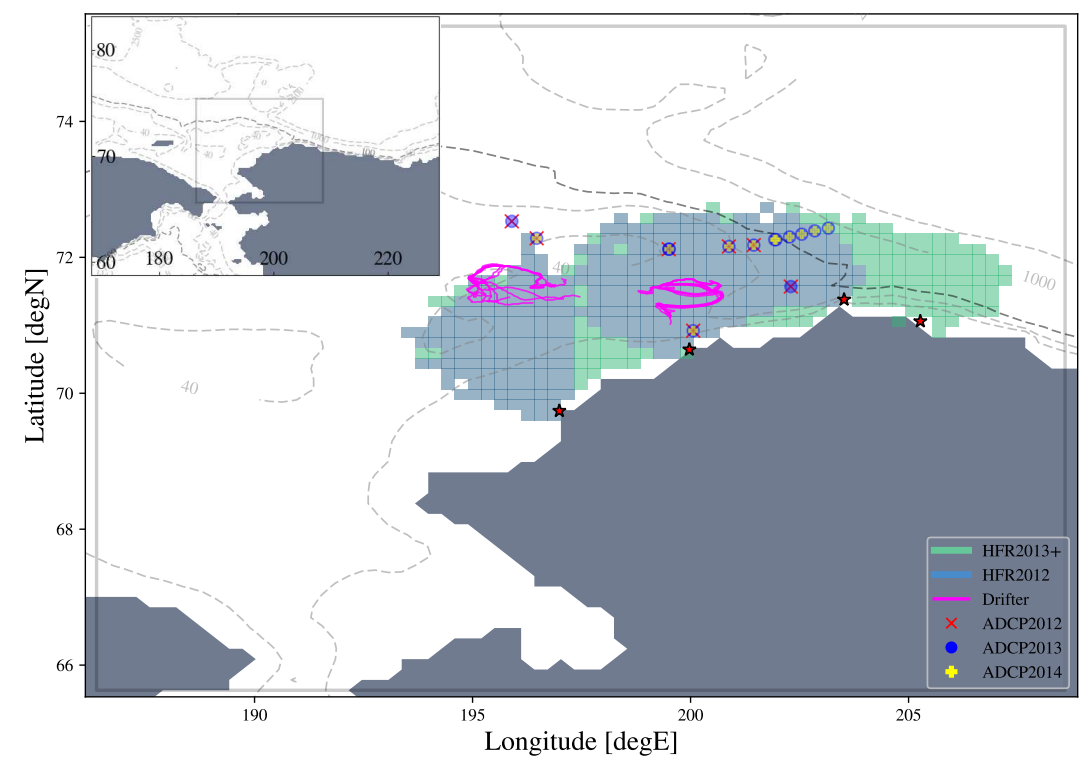

Figure 1: Chukchi domain and observations for 2012-2014 shown focused on subregion resolved at approximately $12 \mathrm{~km}$ bordered in light grey line; the inset image shows the entire domain. Stars identify approximate locations of HFR antennae. 

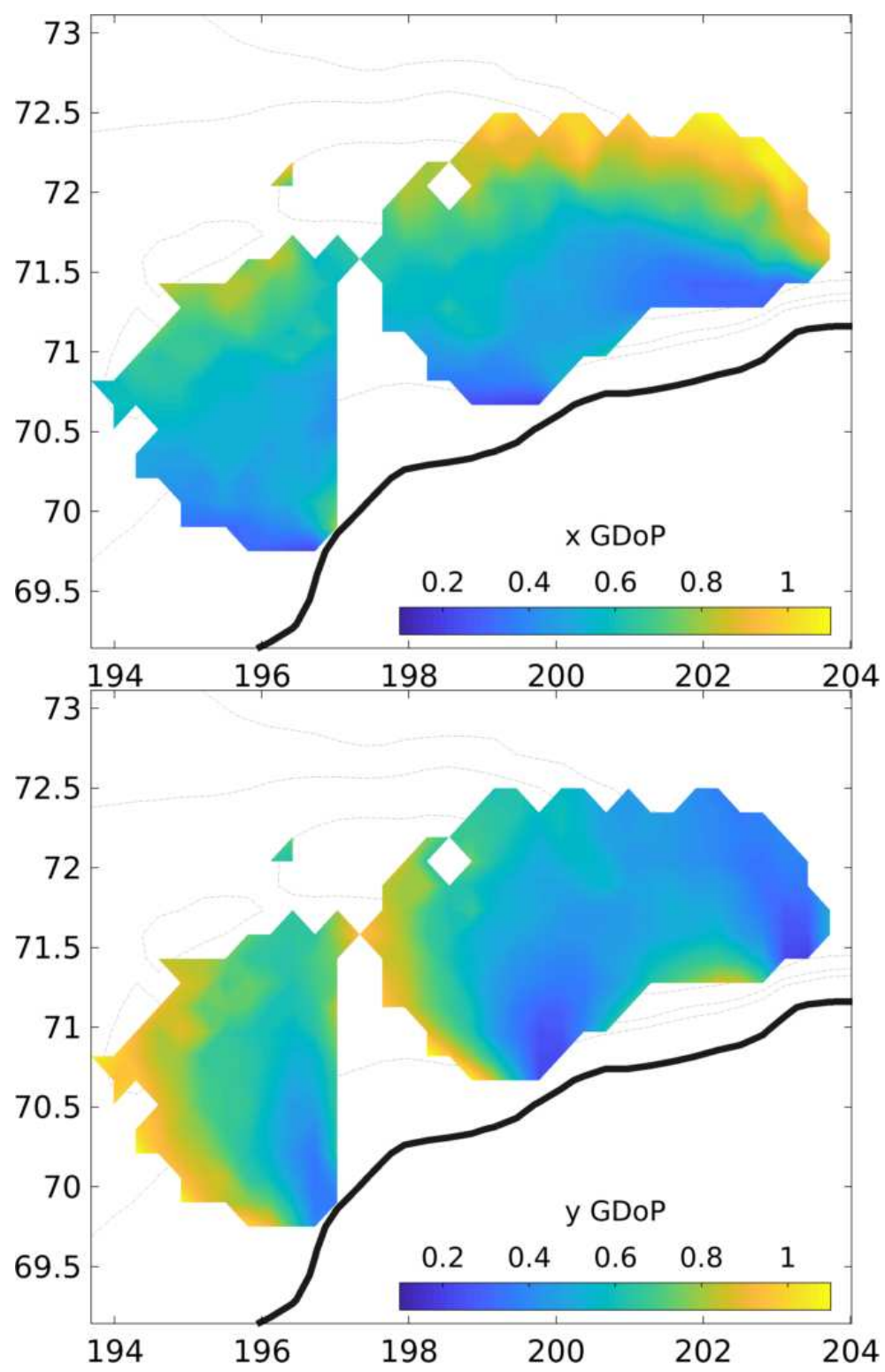

Figure 2: Temporally averaged HFR GDOP fields. Temporally averaged HFR GDOP fields are shown to illustrate the scaling applied to $\sigma_{h}$ to generate the pointwise values in error covariance matrix factor $\mathbf{R}^{1 / 2}$. 
Table 1: Moored ADCP information. The table shows the internal mooring reference number, name in previous studies, geographical location, and deployment/retrieval dates. The names corresponds to Barrow Canyon (BC), Hanna Shoal Northeast/Northwest (HSNE/NW) in Weingartner et al. (2017a), and East and West Barrow Canyon (EBC, WBC). The dates are rounded to the first analysis time with a complete 6-hour record, and date specified as '-' indicates data through 2014-310.

\begin{tabular}{cccccc} 
ID & Name & Lat. $\left({ }^{\circ} \mathrm{N}\right)$ & Lon. $\left({ }^{\circ} \mathrm{E}\right)$ & Start & End \\
\hline Mooring 13 & BC2 & 70.92 & 200.06 & $2012-255.50$ & - \\
Mooring 16 & CS_1 \#01 & 72.26 & 201.93 & $2013-290.50$ & - \\
Mooring 17 & CS_2 \#02 & 72.30 & 202.27 & $2013-287.50$ & - \\
Mooring 18 & CS_3 \#03 & 72.34 & 202.55 & $2013-287.75$ & - \\
Mooring 19 & CS_4 \#04 & 72.39 & 202.85 & $2013-287.75$ & - \\
Mooring 20 & CS_5 \#05 & 72.43 & 203.16 & $2013-287.75$ & - \\
Mooring 21 & FM_1 \#06 & 72.26 & 201.96 & $2013-300.75$ & - \\
Mooring 22 & HS-NE_40m & 72.12 & 199.50 & $2012-236.25$ & - \\
Mooring 23 & HS-NE_50m & 72.16 & 200.88 & $2012-236.50$ & - \\
Mooring 24 & HS-NE_60m & 72.18 & 201.45 & $2012-236.75$ & - \\
Mooring 25 & HS-NW_40m & 72.28 & 196.47 & $2012-231.50$ & - \\
Mooring 26 & HS-NW_50m & 72.53 & 195.90 & $2012-231.25$ & - \\
Mooring 27 & HS-NE_40m & 72.12 & 199.51 & $2013-254.00$ & - \\
Mooring 28 & HS-NE_50m & 72.16 & 200.88 & $2013-254.00$ & - \\
Mooring 29 & HS-NE_60m & 72.18 & 201.45 & $2013-253.75$ & - \\
Mooring 30 & HS-NW_40m & 72.28 & 196.47 & $2013-254.75$ & - \\
Mooring 31 & HS-NW_50m & 72.53 & 195.90 & $2013-254.75$ & - \\
Mooring 34 & EBC & 71.38 & 203.12 & $2011-233.75$ & $2012-245.50$ \\
Mooring 36 & WBC & 71.57 & 202.30 & $2012-286.75$ & $2013-248.75$
\end{tabular}


Table 2: Drifter Information. The table shows the internal drifter reference number, name in previous studies, geographical location, and deployment/termination date. Drifter IDs identify the deployment locations for paths shown in in Figure 1. The names corresponds to the corresponding public data records, available and visualizable at research.cfos . uaf . edu/chukchi-beaufort/data/drifters/ under heading "BOEM 13-August-2012".

\begin{tabular}{cccccc} 
ID & Name & Lat. $\left({ }^{\circ} \mathrm{N}\right)$ & Lon. $\left({ }^{\circ} \mathrm{E}\right)$ & Start & End \\
\hline 72 & UAFSFOS-MS-0001 & 71.628 & 195.277 & $2012-225.42$ & $2012-284.54$ \\
73 & UAFSFOS-MS-0003 & 71.570 & 199.303 & $2012-236.71$ & $2012-261.08$ \\
74 & UAFSFOS-MS-0004 & 71.627 & 195.290 & $2012-225.42$ & $2012-296.12$ \\
75 & UAFSFOS-MS-0005 & 71.628 & 195.280 & $2012-225.42$ & $2012-296.54$ \\
76 & UAFSFOS-MS-0006 & 71.628 & 195.280 & $2012-225.42$ & $2012-285.67$ \\
77 & UAFSFOS-MS-0007 & 71.626 & 195.290 & $2012-225.42$ & $2012-285.88$ \\
78 & UAFSFOS-MS-0008 & 71.568 & 199.301 & $2012-236.71$ & $2012-250.33$ \\
79 & UAFSFOS-MS-0009 & 71.628 & 195.284 & $2012-225.42$ & $2012-259.83$ \\
80 & UAFSFOS-MS-0011 & 71.569 & 199.302 & $2012-236.71$ & $2012-290.00$ \\
81 & UAFSFOS-MS-0012 & 71.569 & 199.304 & $2012-236.71$ & $2012-278.62$ \\
82 & UAFSFOS-SVP-0001 & 71.568 & 199.296 & $2012-236.71$ & $2012-319.17$ \\
83 & UAFSFOS-SVP-0002 & 71.634 & 195.255 & $2012-225.42$ & $2012-317.04$ \\
84 & UAFSFOS-SVP-0003 & 71.634 & 195.264 & $2012-225.42$ & $2012-311.29$ \\
85 & UAFSFOS-SVP-0004 & 71.570 & 199.296 & $2012-236.71$ & $2013-041.83$ \\
86 & UAFSFOS-SVP-0005 & 71.635 & 195.262 & $2012-225.42$ & $2013-041.83$ \\
87 & UAFSFOS-SVP-0006 & 71.634 & 195.255 & $2012-225.42$ & $2013-041.83$ \\
88 & UAFSFOS-SVP-0007 & 71.572 & 199.288 & $2012-236.71$ & $2013-012.08$ \\
89 & UAFSFOS-SVP-0008 & 71.629 & 195.259 & $2012-225.42$ & $2012-332.67$ \\
90 & UAFSFOS-SVP-0009 & 71.573 & 199.296 & $2012-236.71$ & $2012-255.62$ \\
91 & UAFSFOS-SVP-0010 & 71.634 & 195.261 & $2012-225.42$ & $2012-330.71$ \\
92 & UAFSFOS-SVP-0011 & 71.571 & 199.287 & $2012-236.71$ & $2013-041.83$ \\
93 & UAFSFOS-SVP-0012 & 71.577 & 199.283 & $2012-236.71$ & $2013-007.88$
\end{tabular}




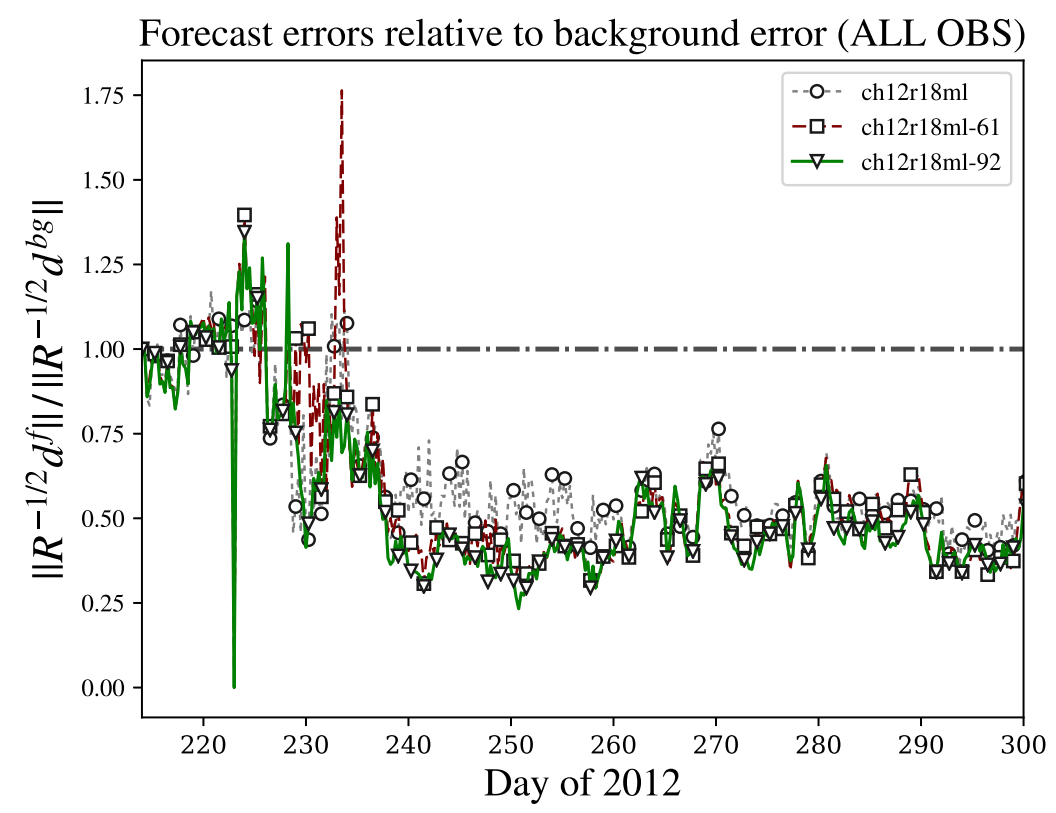

Figure 3: Forecast-minus-observation relative differences for different ensemble size. Varying ensemble-size forecast model errors $\left\|\mathbf{R}^{-1 / 2} d^{f}\right\|$ relative to the background for all observations. The horizontal grey line indicates the covariance-weighted background innovation norm errors $\left\|\mathbf{R}^{-1 / 2} d^{b g}\right\|$ used as a reference. The 30,61 , and 92 element filters are indicated by lines with circle, square, and triangle markers, respectively. 

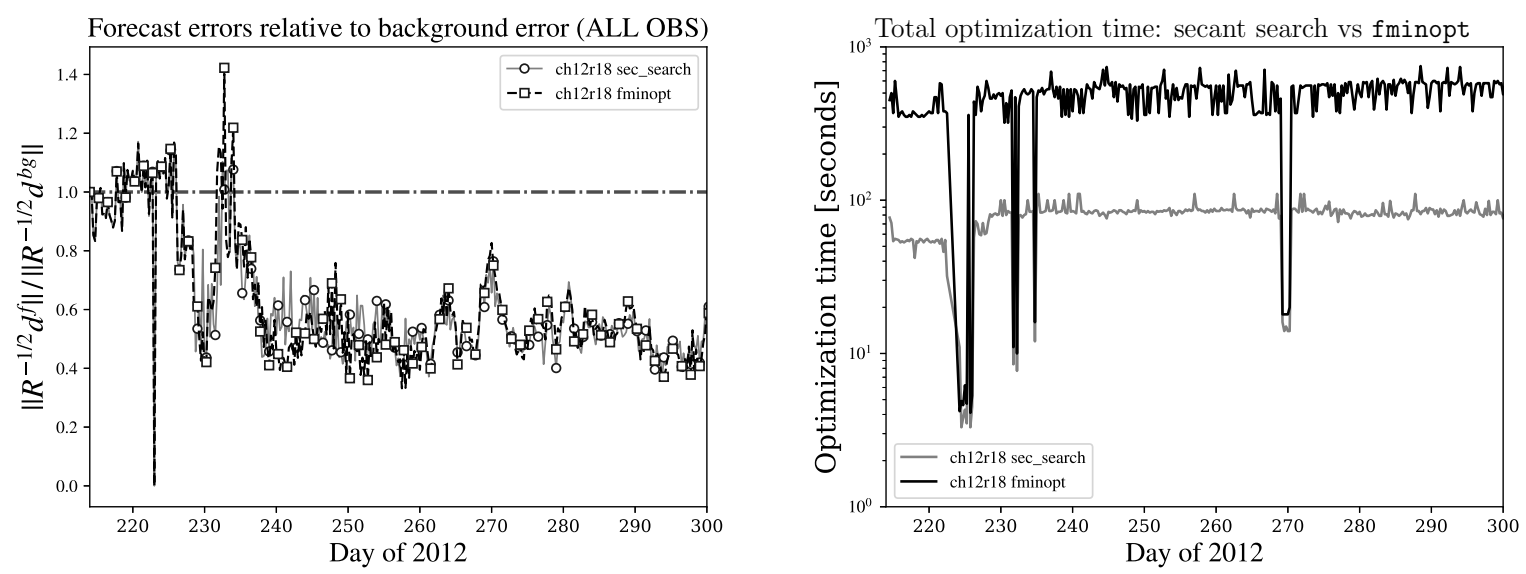

Figure 4: Efficiency of optimization schemes. Time series of relative errors (left) and computation time for optimization (right) via secant-search and "fminopt" algorithms. The right plot suggests that the custom optimization code finds the same optima as the proprietary optimization routine, but does so approximately one order of magnitude ( $\sim 6.5$ times) faster.

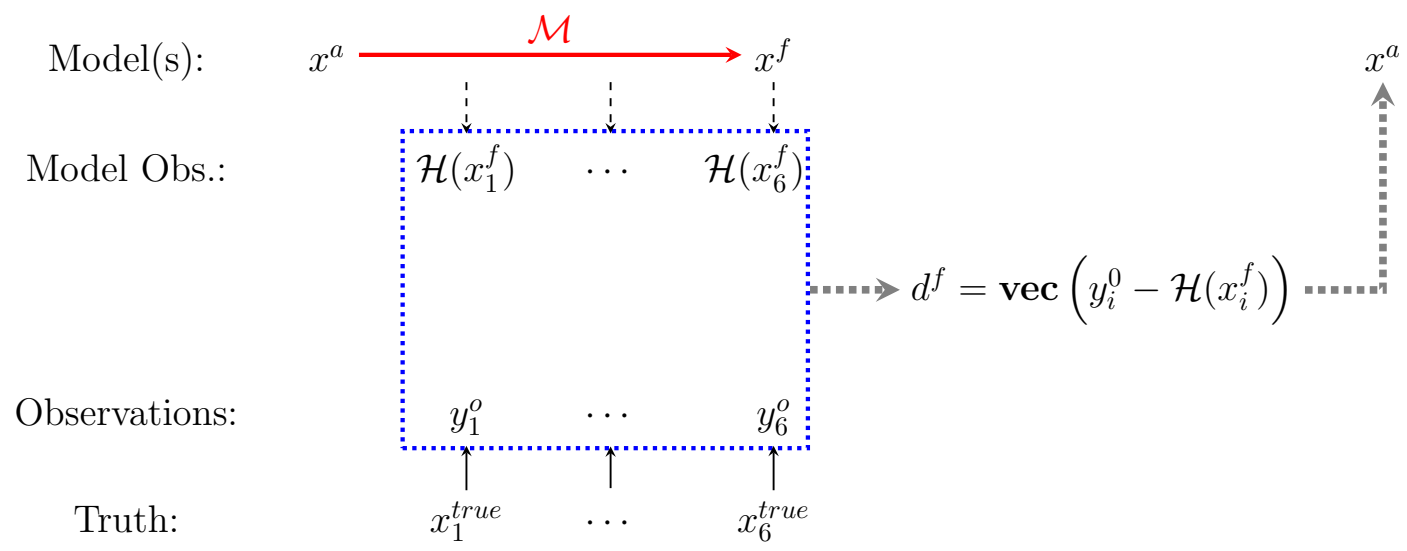

Figure 5: "Asynchronous" Assimilation Process. The conceptual relationship between the true ocean, observational data, modeled data, and model states is shown. The red arrow and application of the nonlinear model comprise the forecast stage. The analysis update uses the comparison of observations shown in the blue box. In the asynchronous case, observations at various times during the forecast stage inform the analysis. 


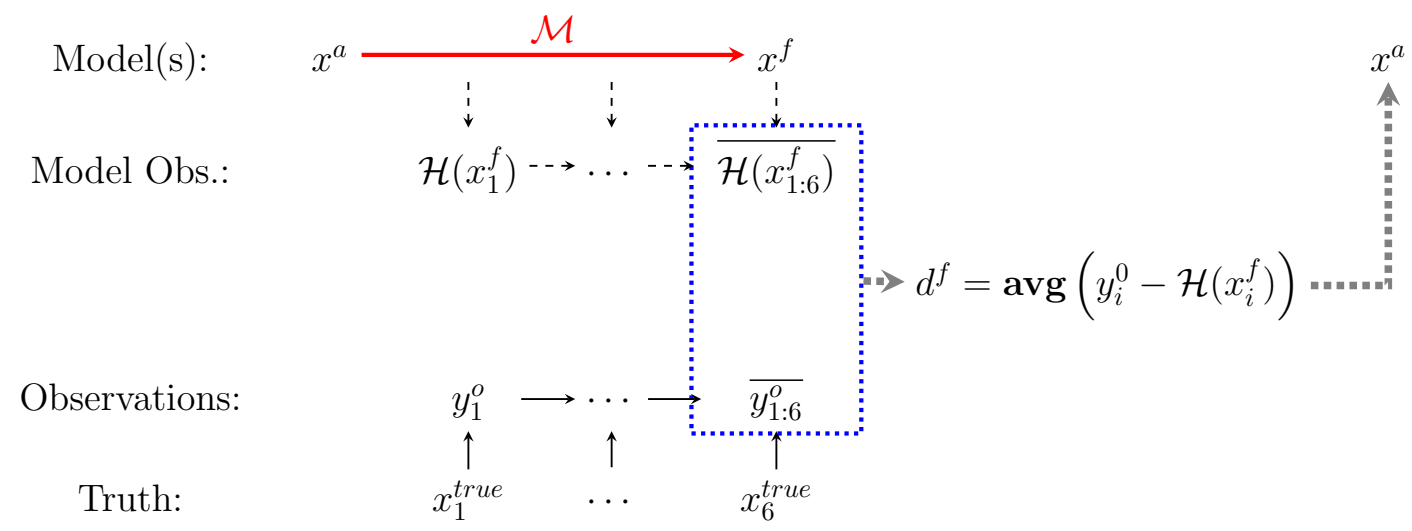

Figure 6: "Synchronous" Assimilation Process. The conceptual relationship between the true ocean, observational data, modeled data, and model states is shown for one case of synchronous observations. In this synchronous case method, observations are represented by averaging HFR over the forecast stage. 


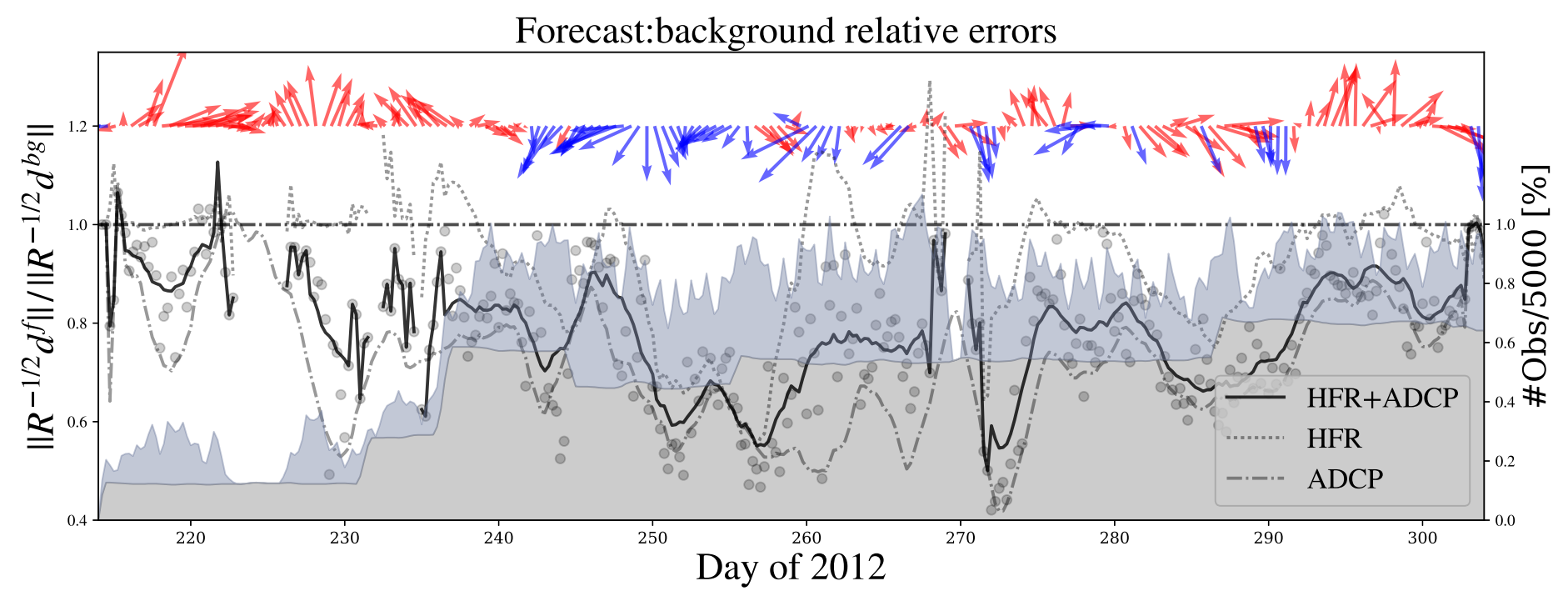

Figure 7: Relative Error Reduction for the 2012 Asynchronous Case. Forecast errors during summer months of 2012 are shown for the case of asynchronously assimilated HFR data. Values are smoothed over 48-hour periods and normalized against the corresponding errors in the background model indicated by the unit horizontal line. Solid black, dotted grey, and dashed grey lines correspond to normalized error values of all observations, HFR observations, and ADCP observations respectively. Pointwise values of total error are shown by grey circles. The local wind forcing vectors in the region are shown at the top of the plot, and assimilated HFR (ADCP) data volume data is shown shaded in blue-gray (beige) for reference. Blue wind vectors denote wind with magnitude greater than $5 \mathrm{~m} / \mathrm{s}$ and blowing toward $225 \pm 60^{\circ}$ (measured counterclockwise from east). 


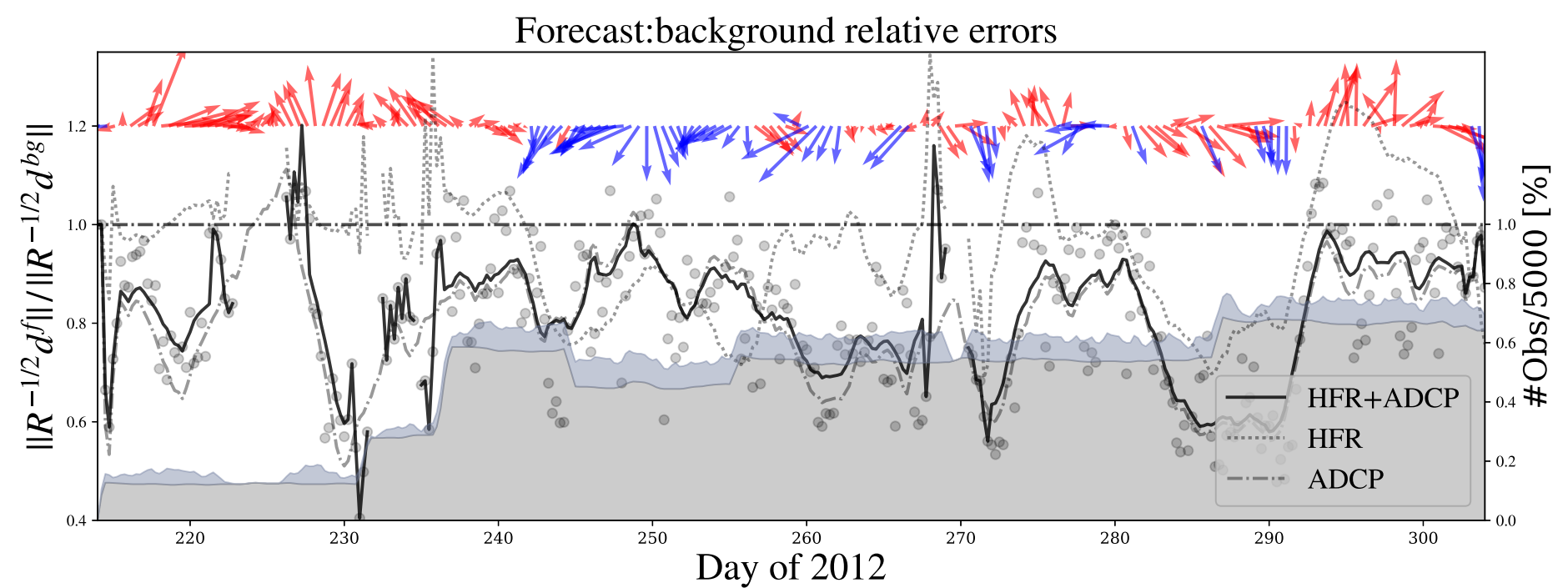

Figure 8: Relative Error Reduction for the 2012 Synchronous Case. Forecast errors for the case of averaged HFR assimilation. Figure layout follows that of Figure 7 and shows results of Case 2 which assimilates mean HFR data. 


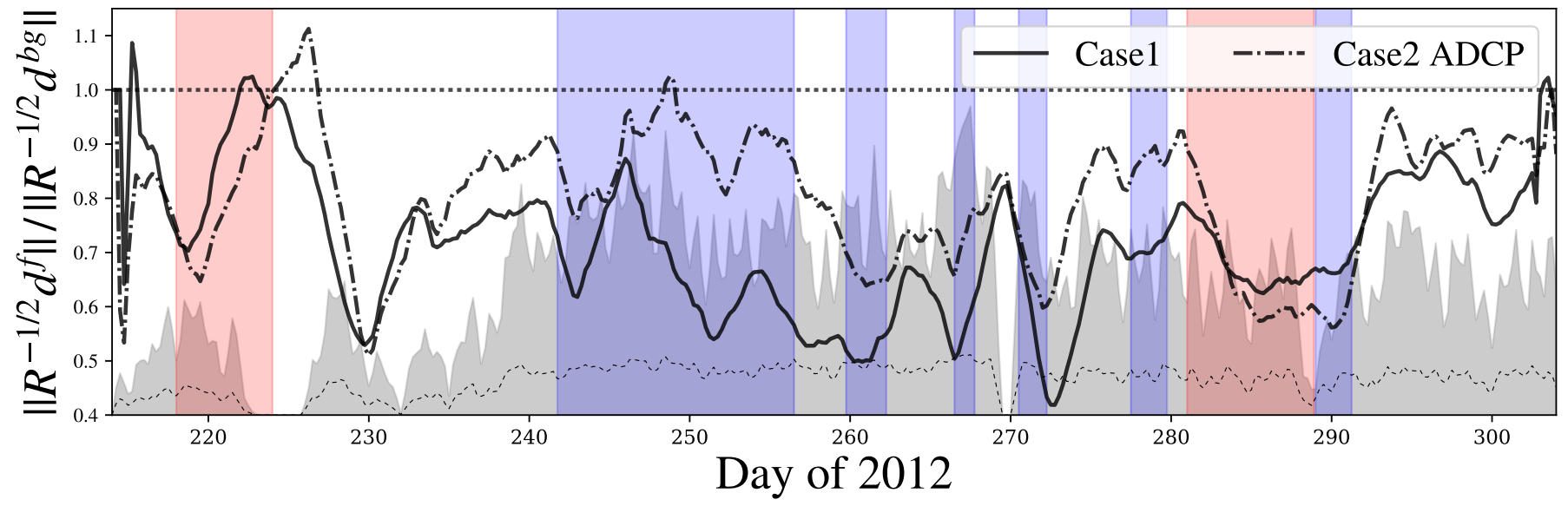

Figure 9: ADCP errors with Identified Wind Regime. The figure shows relative ADCP errors with the solid (dot-dash) line showing Case 1 (Case 2). Normalization with with respect to background errors, as in previous plots. Blue and red regions identify times with "opposing" and "supporting" winds, respectively, as described in the text. The volume of HFR observations for Case 1 is shown in the grey background for reference, with the low, dotted line indicating the volume of averaged HFR observations. 

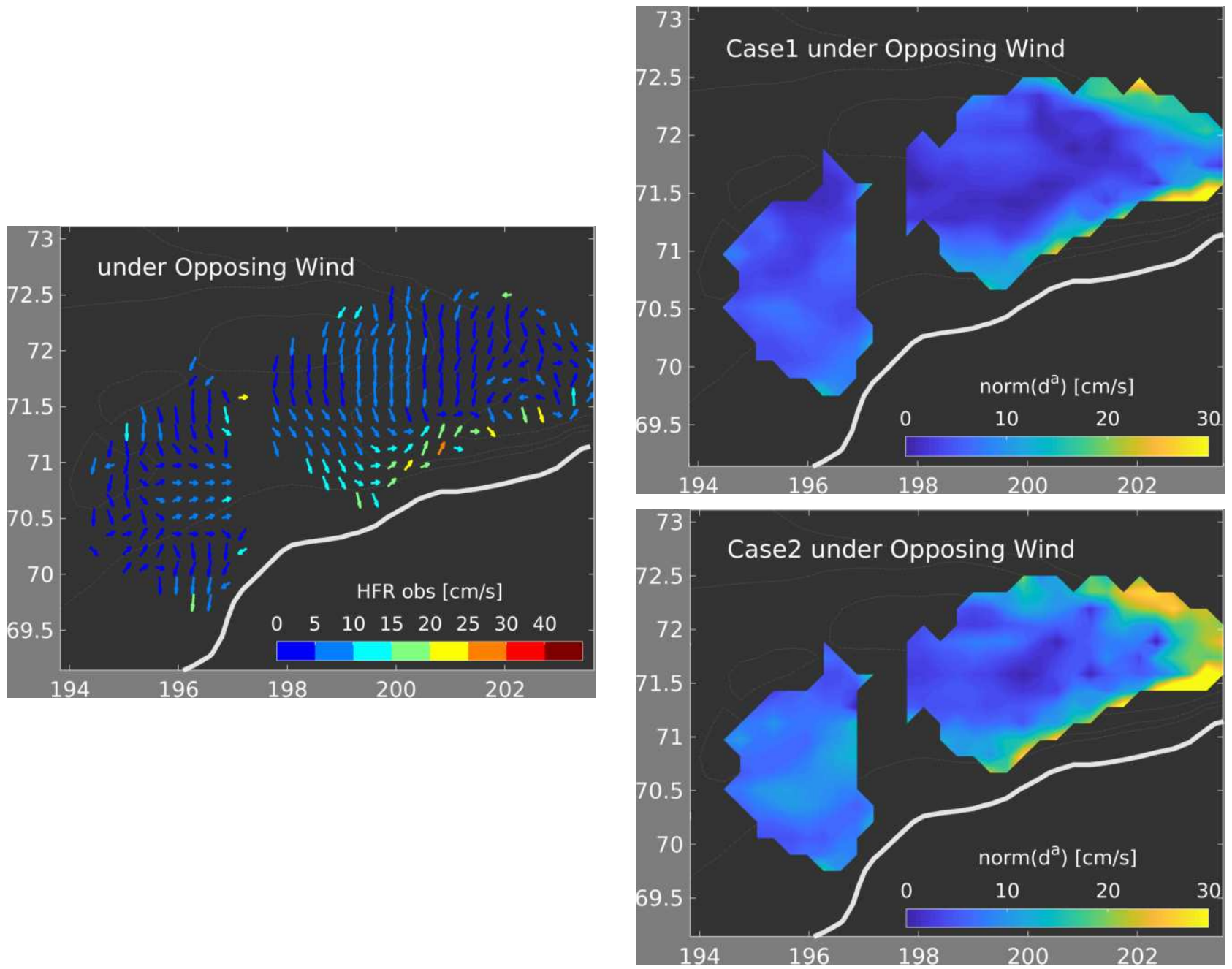

Figure 10: Mean HFR Observations and Analysis Errors under Opposing Winds. Arrows indicate the temporally-averaged HFR observations during opposing wind events in the left panel, with colors indicating magnitude. The corresponding averaged errors for Case 1 and Case 2 are shown in the upper right and lower right panels, respectively. The heavy white line identifies the approximate Alaska coast from the model $3 \mathrm{~m}$ bathymetry. Dotted contours identify the 50, 50, and 70 m model isobaths. 

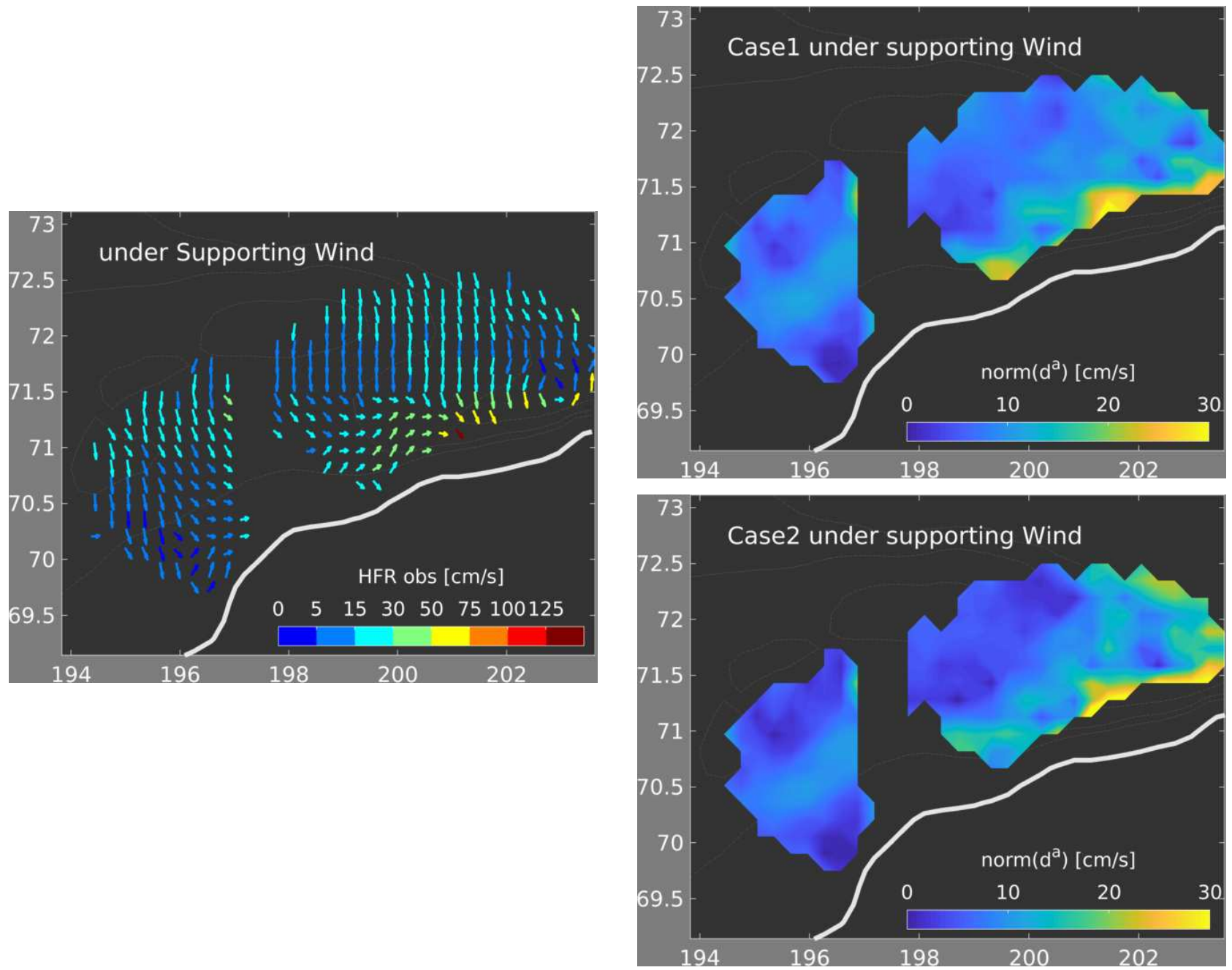

Figure 11: Mean HFR Observations and Analysis Errors under Supporting Winds. The plot layout is identical to that of Figure 10, only for supporting wind events. 


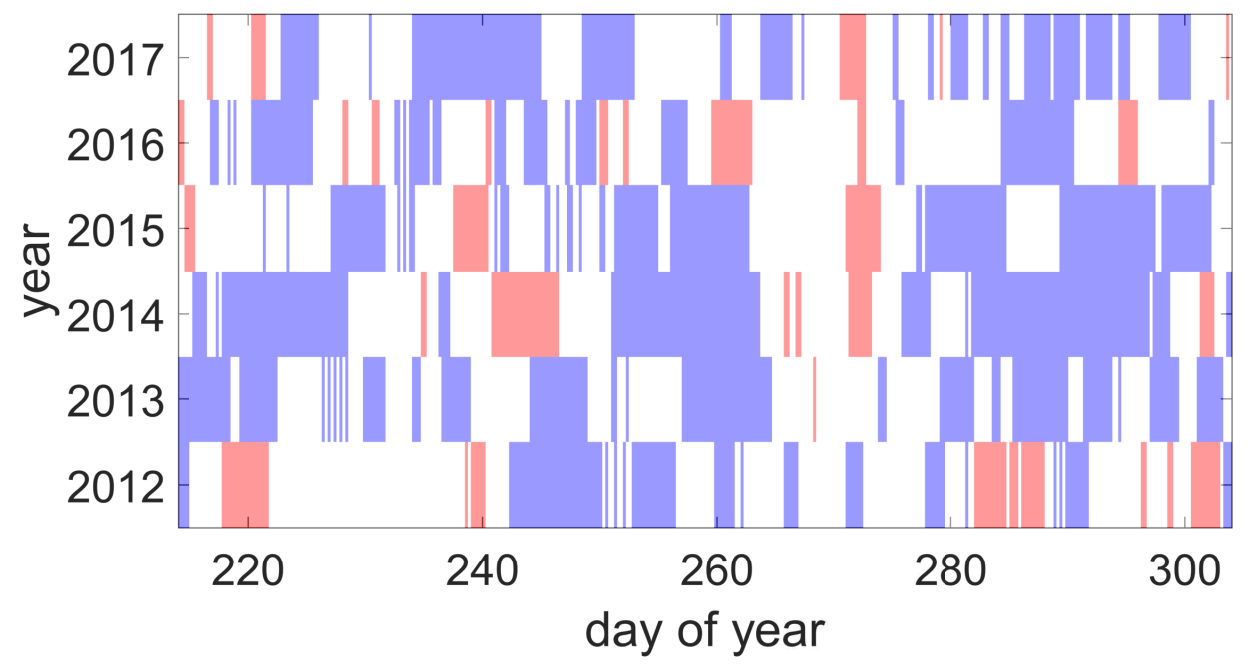

Figure 12: Temporal Map of Wind Regimes for 2012-2017 Summers. Shown in red and blue are the opposing and supporting wind events from spatial means of ERA-Interim 6-hourly 10 $\mathrm{m}$ wind analysis over the $12 \mathrm{~km}$ model region. The criterion used to establish the supporting wind events omits the temporal restrictions.
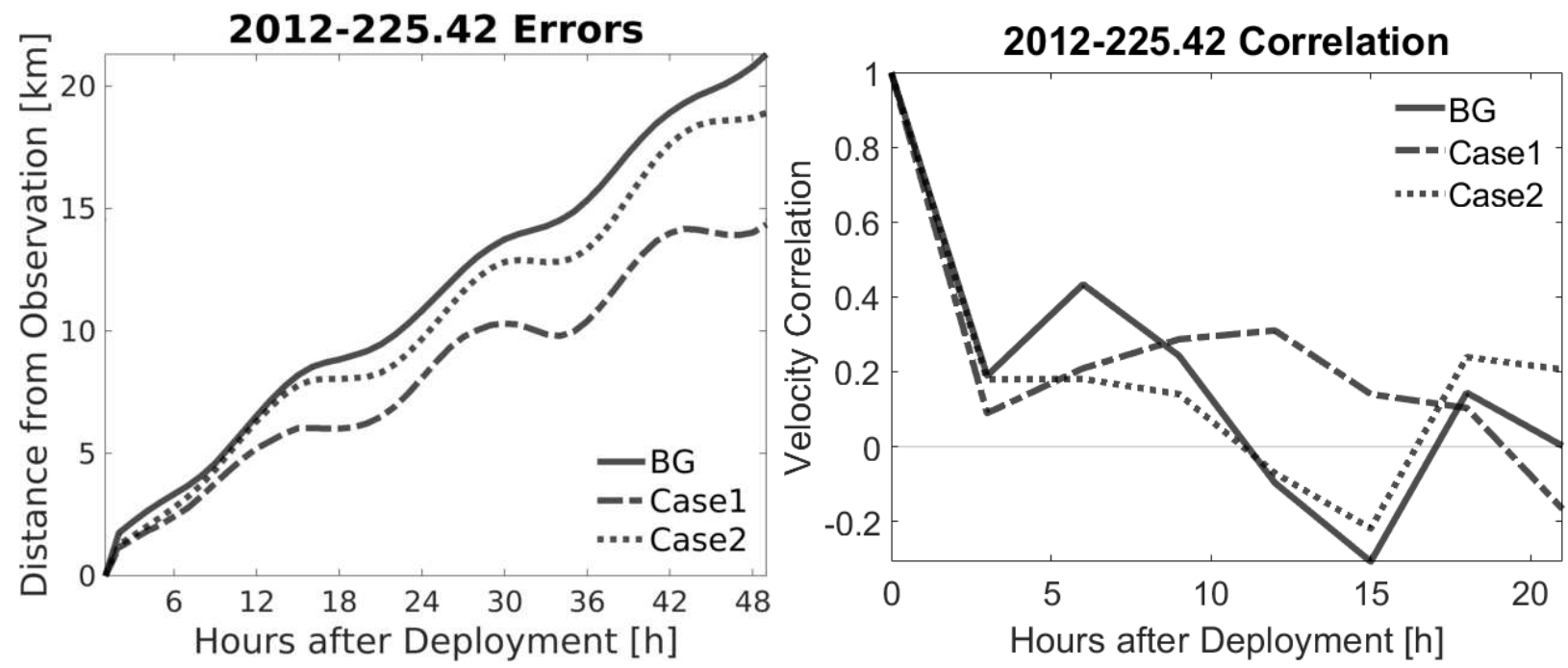

Figure 13: Shoal Region Model-Drifter Position and Velocity Correspondence. Correspondence between forecast and drifters deployed on 2012-225.42 is shown here, with calculated distance from observation in the left panel and timeseries of correlation $r(t)$ in the right panel. 

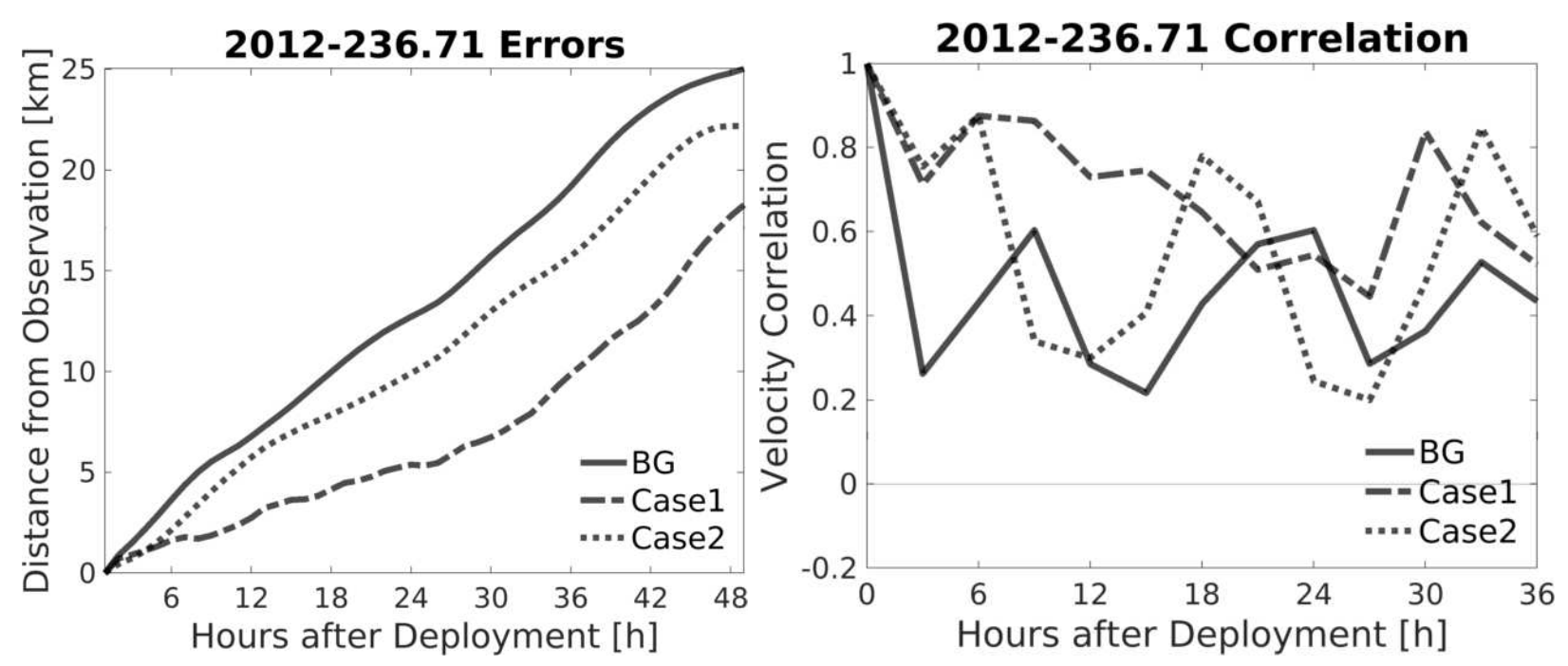

Figure 14: ACC Region Model-Drifter Position and Velocity Correspondence. Correspondence between forecast and drifters deployed on 2012-236.71 is shown, with the panels presented as in Figure 14. 


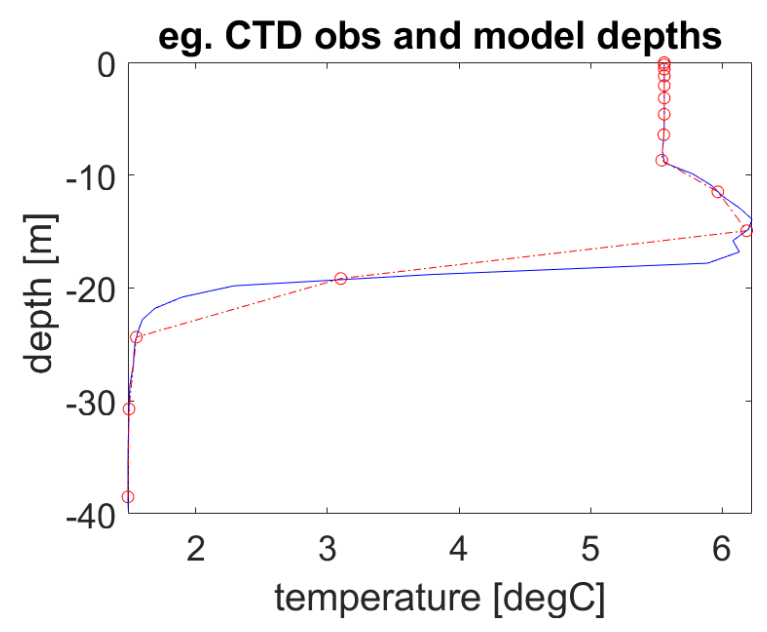

Figure 15: Example CTD observation and model representative. The blue curve shows temperature plotted against depth as represented in observational data. The projection onto modeled vertical coordinates using cubic spline interpolation is shown by the dashed red curve, with circles indicating values at ROMS vertical coordinate depths. 


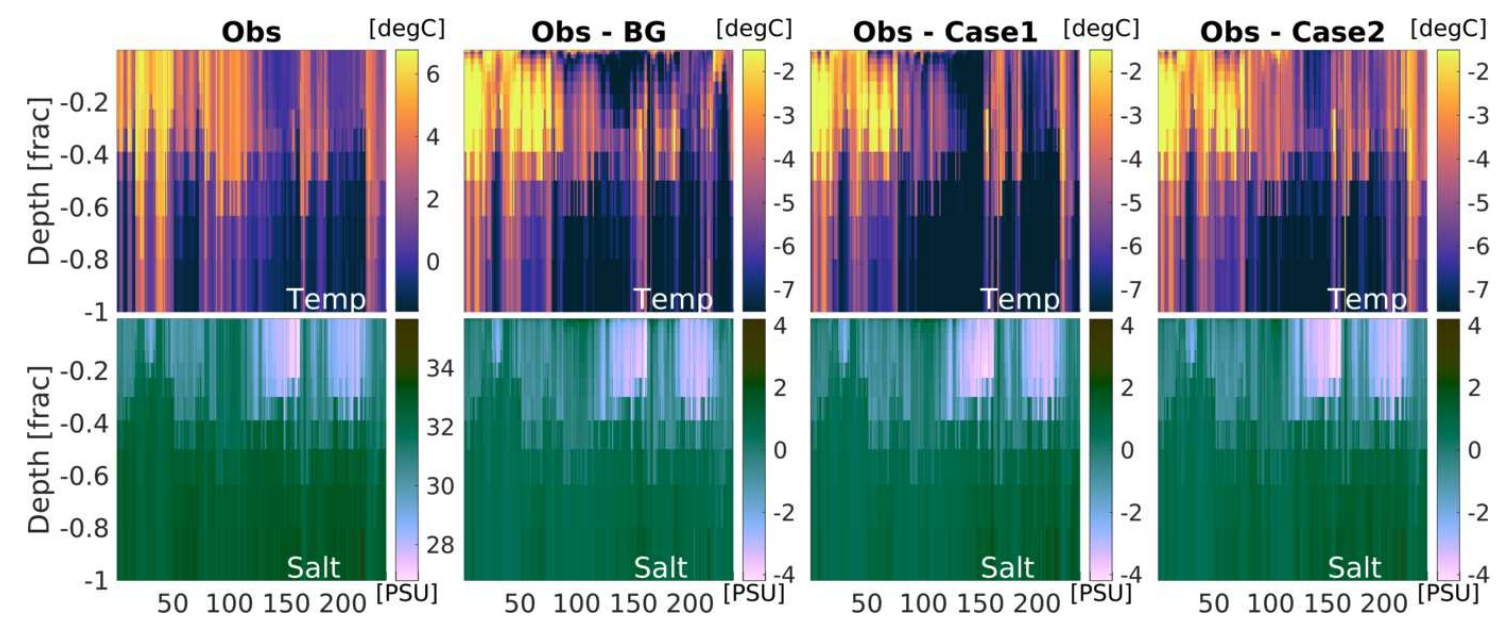

Figure 16: CTD Observations and associated Model Errors. The top row of panels shows temperatures and the bottom row shows salinities where the horizontal axes correspond to chronologically sorted CTD observations and the vertical axes to fraction of total depth. The four columns, left to right, show CTD observations and associated errors for the background model, Case 1, and Case 2, respectively. The horizontal axis limits correspond roughly to 2012-230-270, although the spacing is not uniform.
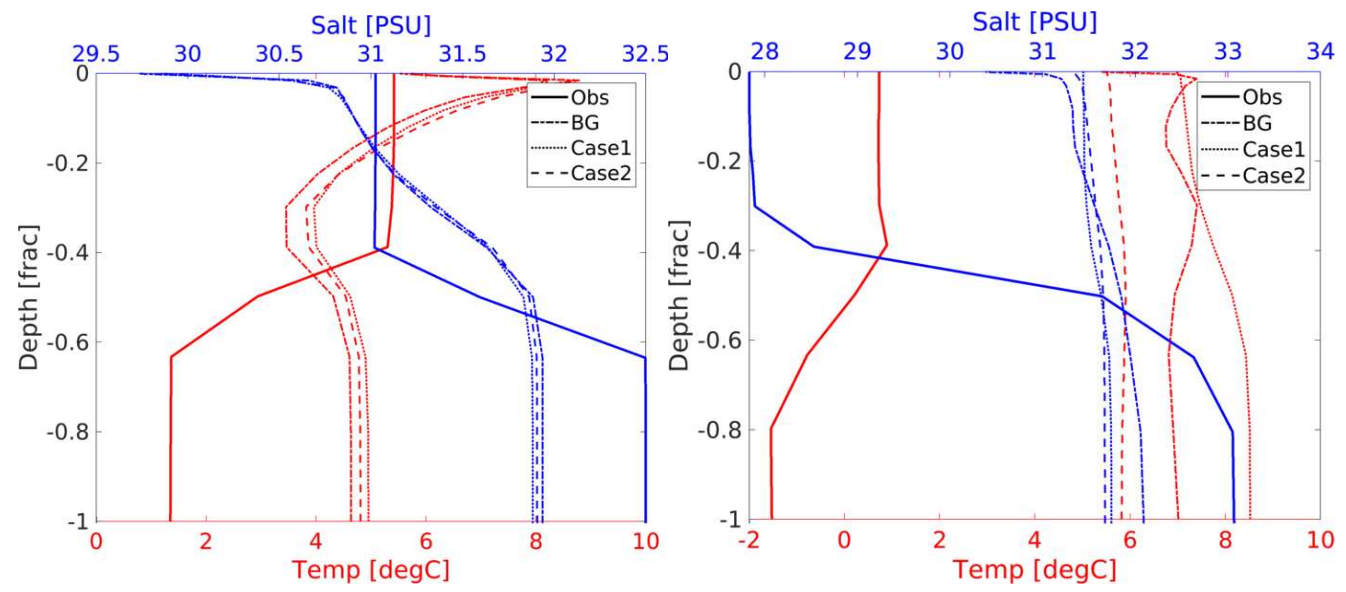

Figure 17: Two Example CTD and model T/S Profiles. The various temperature (red) and salinity (blue) profiles associated with CTD observations at $\sim j d 230$ (left) and jd270. CTD data, background forecast, Case 1 forecast, and Case 2 forecasts are shown by solid, dash-dot, dotted, and dashed lines, respectively. The difference is extreme, but illustrates the model T/S drift toward strongly biased uniform profiles. 


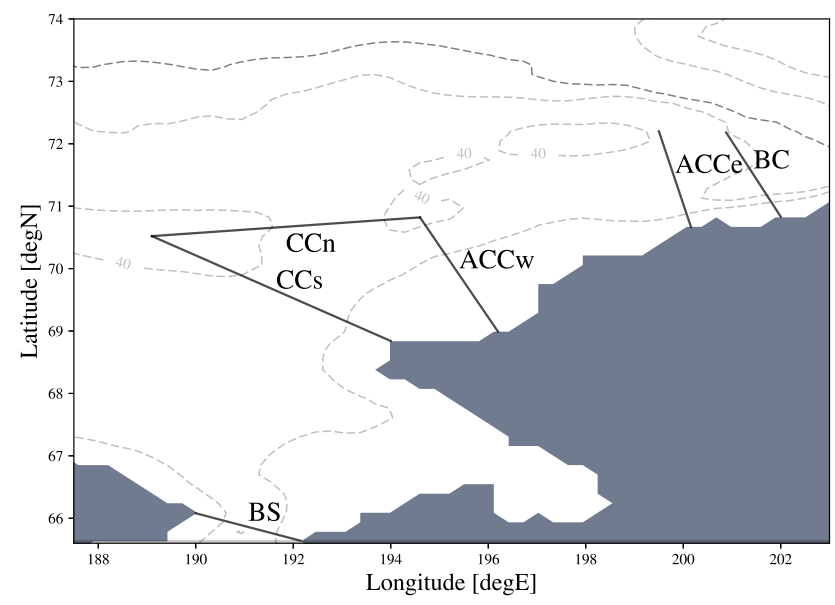

Figure 18: Map of Model Transects. The geographical locations of sections used for estimation of transports are shown with corresponding short identification labels for transects. Long Strait (LS) is far west of the region and is meridionally aligned at $178.8^{\circ}$ between Wrangell and the Siberian coast. Labels are shown on the positively oriented side of each segment. 


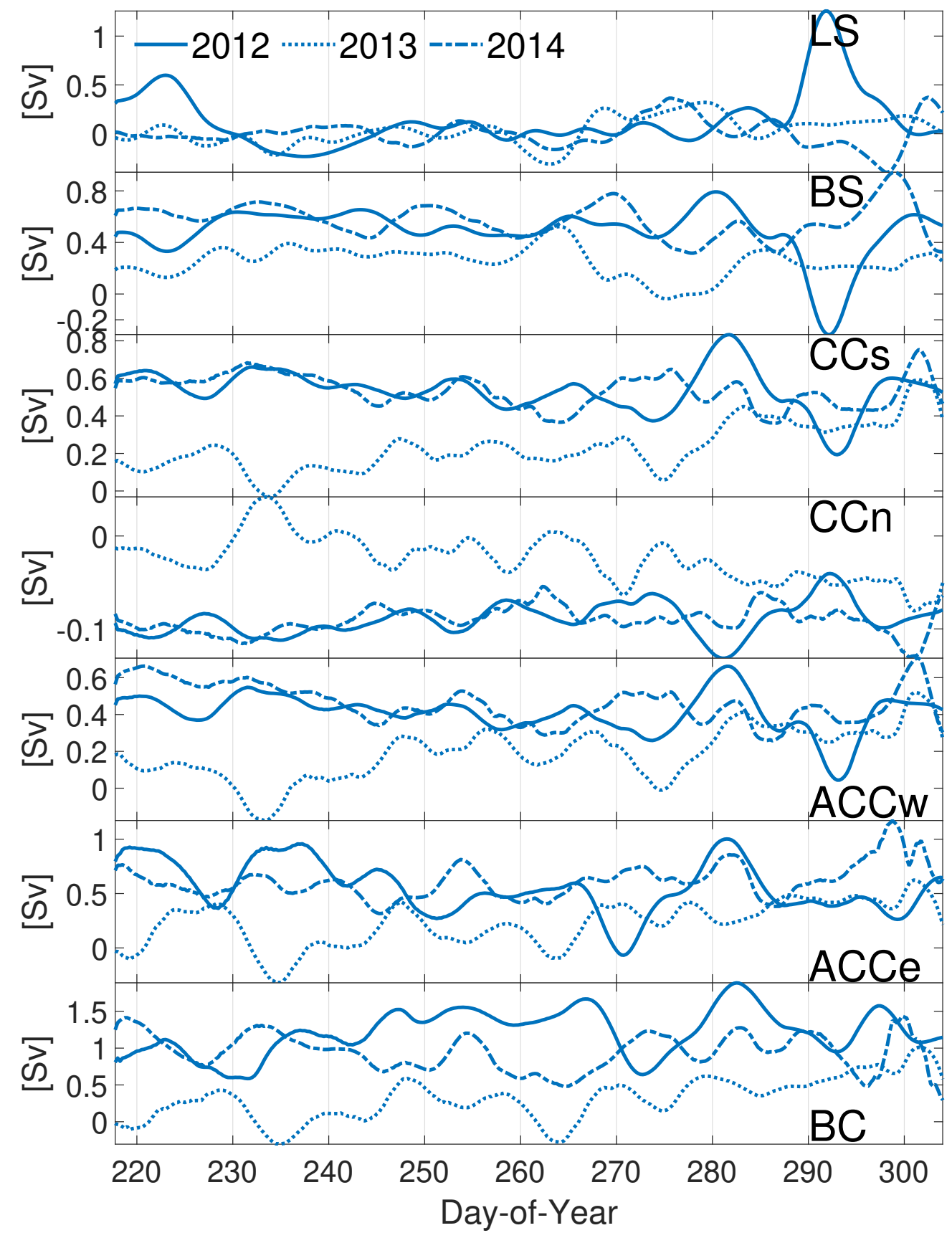

Figure 19: Seasonal Mass Transport Estimates 2012-2014. Mass transport estimates during 2012-2014 are shown for the various geographical transects. Plots of 2013 transport across the central channel and eastern coastal region look qualitatively different than 2012 and 2014 reconstructions. Plotted data is smoothed over 5-day intervals for presentability, while figures stated in the text use daily averages. 


\section{References}

Ashkenazy, Y., and H. Gildor (2011), On the probability and spatial distribution of ocean surface currents, Journal of Physical Oceanography, 41(12), 2295-2306.

Awaji, T., S. Masuda, Y. Ishikawa, N. Sugiura, T. Toyoda, and T. Nakamura (2003), State estimation of the North Pacific Ocean by a four-dimensional variational data assimilation experiment, Journal of Oceanography, 59(6), 931-943.

Barth, A., J.-M. Beckers, A. Alvera-Azcárate, and R. H. Weisberg (2007), Filtering inertiagravity waves from the initial conditions of the linear shallow water equations, Ocean Modelling, 19(3), 204-218.

Bracco, A., E. P. Chassignet, Z. D. Garraffo, and A. Provenzale (2003), Lagrangian velocity distributions in a high-resolution numerical simulation of the North Atlantic, Journal of Atmospheric and Oceanic Technology, 20(8), 1212-1220.

Brasseur, P. P., and J. A. Haus (1991), Application of a 3-D variational inverse model to the analysis of ecohydrodynamic data in the Northern Bering and Southern Chukchi Seas, Journal of Marine Systems, 1(4), 383-401.

Brugler, E. T., R. S. Pickart, G. Moore, S. Roberts, T. J. Weingartner, and H. Statscewich (2014), Seasonal to interannual variability of the Pacific water boundary current in the Beaufort Sea, Progress in Oceanography, 127, 1-20.

Burgers, G., P. Jan van Leeuwen, and G. Evensen (1998), Analysis Scheme in the Ensemble Kalman Filter, Monthly Weather Review, 126(6), 1719-1724, doi:10.1175/1520-0493(1998) 126〈1719:ASITEK $\rangle 2.0 . \mathrm{CO} ; 2$.

Chapman, R., L. Shay, H. Graber, J. Edson, A. Karachintsev, C. Trump, and D. Ross (1997), On the accuracy of HF radar surface current measurements: Intercomparisons with shipbased sensors, Journal of Geophysical Research: Oceans, 102(C8), 18,737-18,748. 
Chepurin, G. A., J. A. Carton, and D. Dee (2005), Forecast model bias correction in ocean data assimilation, Monthly Weather Review, 133, 1328-1342, doi:10.1175/MWR2920.1.

Coachman, L. K., L. K. Coachman, K. Aagaard, and R. Tripp (1975), Bering Strait: the regional physical oceanography, University of Washington Press.

Corlett, W. B., and R. S. Pickart (2017), The Chukchi slope current, Progress in Oceanography, 153, 50-65, doi:10.1016/j.pocean.2017.04.005.

Danielson, S. L., T. J. Weingartner, K. S. Hedstrom, K. Aagaard, R. Woodgate, E. Curchitser, and P. J. Stabeno (2014), Coupled wind-forced controls of the Bering-Chukchi shelf circulation and the Bering Strait throughflow: Ekman transport, continental shelf waves, and variations of the Pacific-Arctic sea surface height gradient, Progress in Oceanography, 125, 40-61, doi:10.1016/j.pocean.2014.04.006.

Danielson, S. L., E. L. Dobbins, M. Jakobsson, M. A. Johnson, T. Weingartner, W. Williams, and Y. Zarayskaya (2015), Sounding the northern seas, Eos, 96.

Davis, R. E. (1985), Drifter observations of coastal surface currents during code: The statistical and dynamical views, Journal of Geophysical Research: Oceans, 90(C3), 4756-4772.

Dee, D. P. (2005), Bias and data assimilation, Quarterly Journal of the Royal Meteorological Society, 131 (613), 3323-3343, doi:10.1256/qj.05.137.

Dee, D. P., and A. M. Da Silva (1998), Data assimilation in the presence of forecast bias, Quarterly Journal of the Royal Meteorological Society, 124(545), 269-295.

ECMWF (2012), Era-interim project, single parameter 6-hourly surface analysis and surface forecast time series.

Evensen, G. (2003), The ensemble Kalman filter: Theoretical formulation and practical implementation, Ocean dynamics, 53(4), 343-367. 
Francis, O. P., M. Yaremchuk, G. G. Panteleev, J. Zhang, and M. Kulakov (2017), Anomalous circulation in the Pacific sector of the Arctic Ocean in July-December 2008, Ocean Modelling, 117, 12-27.

Gong, D., and R. S. Pickart (2015), Summertime circulation in the eastern Chukchi Sea, Deep Sea Research Part II: Topical Studies in Oceanography, 118, 18-31.

Gustafsson, N. (2007), Discussion on 4D-Var or EnKF?, Tellus A, 59(5), 774-777.

Houtekamer, P. L., and H. L. Mitchell (1998), Data assimilation using an ensemble Kalman filter technique, Monthly Weather Review, 126(3), 796-811.

Ide, K., P. Courtier, M. Ghil, and A. C. Lorenc (1997), Unified Notation for Data Assimilation: Operational, Sequential and Variational (<Special Issue $>$ Data Assimilation in Meteorology and Oceanography: Theory and Practice), Journal of the Meteorological Society of Japan. Ser. II, 75 (1B), 181-189.

Itoh, M., K. Shimada, T. Kamoshida, F. McLaughlin, E. Carmack, and S. Nishino (2012), Interannual variability of Pacific Winter Water inflow through Barrow Canyon from 2000 to 2006, Journal of Oceanography, 68(4), 575-592, doi:10.1007/s10872-012-0120-1.

Itoh, M., S. Nishino, Y. Kawaguchi, and T. Kikuchi (2013), Barrow Canyon volume, heat, and freshwater fluxes revealed by long-term mooring observations between 2000 and 2008, Journal of Geophysical Research: Oceans, 118(9), 4363-4379.

Jazwinski, A. H. (2007), Stochastic processes and filtering theory, Courier Corporation.

Kalnay, E. (2003), Atmospheric modeling, data assimilation and predictability, Cambridge University Press.

Kim, S., R. Samelson, and C. Snyder (2009), Ensemble-based estimates of the predictability of wind-driven coastal ocean flow over topography, Monthly Weather Review, 137(8), 2515-2537. 
Le Dimet, F.-X., and O. Talagrand (1986), Variational algorithms for analysis and assimilation of meteorological observations: theoretical aspects, Tellus A: Dynamic Meteorology and Oceanography, 38(2), 97-110.

Luchin, V., and G. Panteleev (2014), Thermal regimes in the Chukchi Sea from 1941 to 2008, Deep Sea Research Part II: Topical Studies in Oceanography, 109, 14-26.

Navon, I., and D. M. Legler (1987), Conjugate-gradient methods for large-scale minimization in meteorology, Monthly Weather Review, 115(8), 1479-1502.

Okkonen, S. R., C. J. Ashjian, R. G. Campbell, W. Maslowski, J. L. Clement-Kinney, and R. Potter (2009), Intrusion of warm Bering/Chukchi waters onto the shelf in the western Beaufort Sea, Journal of Geophysical Research: Oceans, 114(C1).

Panteleev, G., M. Yaremchuk, and D. Nechaev (2009), Optimization of mooring observations in Northern Bering Sea, Dynamics of Atmospheres and Oceans, 48(1-3), 143-154.

Panteleev, G., D. A. Nechaev, A. Proshutinsky, R. Woodgate, and J. Zhang (2010), Reconstruction and analysis of the chukchi sea circulation in 1990-1991, Journal of Geophysical Research: Oceans, $115(\mathrm{C} 8)$.

Panteleev, G., M. Yaremchuk, P. J. Stabeno, V. Luchin, D. A. Nechaev, and T. Kikuchi (2011), Dynamic topography of the Bering Sea, Journal of Geophysical Research: Oceans, $116(\mathrm{C} 5)$.

Panteleev, G., M. Yaremchuk, V. Luchin, D. Nechaev, and T. Kukuchi (2012), Variability of the Bering Sea circulation in the period 1992-2010, Journal of oceanography, 68(4), $485-496$.

Panteleev, G., M. Yaremchuk, O. Francis, and T. Kikuchi (2013), Configuring high frequency radar observations in the Southern Chukchi Sea, Polar Science, 7(2), 72-81. 
Panteleev, G., M. Yaremchuk, J. Stroh, P. Posey, D. Hebert, and D. A. Nechaev (2015), Optimization of the High-Frequency Radar Sites in the Bering Strait Region, Journal of Atmospheric and Oceanic Technology, 32(2), 297-309.

Peralta-Ferriz, C., and R. A. Woodgate (2017), The dominant role of the East Siberian Sea in driving the oceanic flow through the Bering Strait-Conclusions from GRACE ocean mass satellite data and in situ mooring observations between 2002 and 2016, Geophysical Research Letters, $44(22)$.

Pickart, R. S., L. J. Pratt, D. J. Torres, T. E. Whitledge, A. Y. Proshutinsky, K. Aagaard, T. A. Agnew, G. W. K. Moore, and H. J. Dail (2010), Evolution and dynamics of the flow through Herald Canyon in the western Chukchi Sea, Deep Sea Research Part II: Topical Studies in Oceanography, 57(1), 5-26.

Pickart, R. S., G. W. Moore, C. Mao, F. Bahr, C. Nobre, and T. J. Weingartner (2016), Circulation of winter water on the Chukchi shelf in early Summer, Deep-Sea Research Part II: Topical Studies in Oceanography, 130, doi:10.1016/j.dsr2.2016.05.001.

Pires, C. A., O. Talagrand, and M. Bocquet (2010), Diagnosis and impacts of non-gaussianity of innovations in data assimilation, Physica D: Nonlinear Phenomena, 239(17), 1701-1717.

Pisareva, M. N., R. S. Pickart, M. A. Spall, C. Nobre, D. J. Torres, G. W. Moore, and T. E. Whitledge (2015), Flow of pacific water in the western Chukchi Sea: Results from the 2009 RUSALCA expedition, Deep-Sea Research Part I: Oceanographic Research Papers, 105, doi:10.1016/j.dsr.2015.08.011.

Potter, R., T. Weingartner, D. Elizabeth, H. Statscewich, and P. Winsor (2014), Surface circulation patterns in the northeastern chukchi sea, in Alaska Marine Science Symposium.

Purser, R. J. (1984), A new approach to the optimal assimilation of meteorological data by iterative bayesian analysis, in Conference on Weather Forecasting and Analysis, 10 th, Clearwater Beach, FL, pp. 102-105. 
Purser, R. J., and D. F. Parrish (2003), A Bayesian technique for estimating continuously varying statistical parameters of a variational assimilation, Meteorology and Atmospheric Physics, 82(1-4), 209-226, doi:10.1007/s00703-001-0583-x.

Sakov, P., and M. Bocquet (2018), Asynchronous data assimilation with the EnKF in presence of additive model error, Tellus A: Dynamic Meteorology and Oceanography, 70(1), 1414,545 .

Sakov, P., G. Evensen, and L. Bertino (2010), Asynchronous data assimilation with the EnKF, Tellus, Series A: Dynamic Meteorology and Oceanography, 62(1), 24-29, doi:10. 1111/j.1600-0870.2009.00417.x.

Shchepetkin, A. F., and J. C. McWilliams (2005), The regional oceanic modeling system (roms): a split-explicit, free-surface, topography-following-coordinate oceanic model, Ocean modelling, 9(4), 347-404.

Sorenson, H. W. (1970), Least-squares estimation: from Gauss to Kalman, IEEE spectrum, $7(7), 63-68$.

Stewart, R. H., and J. W. Joy (1974), HF radio measurements of surface currents, in Deep Sea Research and Oceanographic Abstracts, vol. 21, pp. 1039-1049, Elsevier.

Talagrand, O. (2003), Bayesian estimation. optimal interpolation. statistical linear estimation, in Data Assimilation for the Earth System, pp. 21-35, Springer.

Teague, C. C., J. F. Vesecky, and Z. R. Hallock (2001), A comparison of multifrequency HF radar and ADCP measurements of near-surface currents during COPE-3, IEEE Journal of Oceanic Engineering, 26(3), 399-405.

Thacker, W. C. (1989), The role of the Hessian matrix in fitting models to measurements, Journal of Geophysical Research: Oceans, 94(C5), 6177-6196. 
Weingartner, T., K. Aagaard, R. Woodgate, S. Danielson, Y. Sasaki, and D. Cavalieri (2005), Circulation on the north central Chukchi Sea shelf, Deep Sea Research Part II: Topical Studies in Oceanography, 52(24), 3150-3174.

Weingartner, T., E. Dobbins, S. Danielson, P. Winsor, R. Potter, and H. Statscewich (2013), Hydrographic variability over the northeastern Chukchi Sea shelf in summer-fall 20082010, Continental Shelf Research, 67, 5-22.

Weingartner, T., Y. C. Fang, P. Winsor, E. Dobbins, R. Potter, H. Statscewich, T. Mudge, B. Irving, L. Sousa, and K. Borg (2017a), The summer hydrographic structure of the Hanna Shoal region on the northeastern Chukchi Sea shelf: 2011-2013, Deep-Sea Research Part II: Topical Studies in Oceanography, 144(August), 6-20, doi:10.1016/j.dsr2.2017.08.006.

Weingartner, T. J., D. J. Cavalieri, K. Aagaard, and Y. Sasaki (1998), Circulation, dense water formation, and outflow on the northeast Chukchi Sea shelf, Journal of Geophysical Research, $103(\mathrm{C} 4), 7647-7661$.

Weingartner, T. J., R. A. Potter, C. A. Stoudt, E. L. Dobbins, H. Statscewich, P. R. Winsor, T. D. Mudge, and K. Borg (2017b), Transport and thermohaline variability in barrow canyon on the northeastern chukchi sea shelf, Journal of Geophysical Research: Oceans, 122(5), 3565-3585.

Wikle, C. K., and L. M. Berliner (2007), A Bayesian tutorial for data assimilation, Physica D: Nonlinear Phenomena, 230(1-2), 1-16, doi:10.1016/j.physd.2006.09.017.

Williams, W. J., E. Shroyer, J. C. Kinney, M. Itoh, and W. Maslowski (2014), Shelf-break exchange in the Bering, Chukchi and Beaufort Seas, in The Pacific Arctic Region, pp. 133-165, Springer.

Winsor, P., and D. C. Chapman (2004), Pathways of Pacific water across the Chukchi Sea: A numerical model study, Journal of Geophysical Research: Oceans, 109(C3). 
Woodgate, R. A. (2018), Increases in the Pacific inflow to the Arctic from 1990 to 2015, and insights into seasonal trends and driving mechanisms from year-round Bering Strait mooring data, Progress in Oceanography, 160, 124-154.

Woodgate, R. A., K. Aagaard, and T. J. Weingartner (2005), Monthly temperature, salinity, and transport variability of the Bering Strait through flow, Geophysical Research Letters, 32(4).

Woodgate, R. A., K. M. Stafford, and F. G. Prahl (2015), A synthesis of year-round interdisciplinary mooring measurements in the Bering Strait (1990-2014) and the RUSALCA years (2004-2011), Oceanography, 28(3), 46-67.

Wright, S., and J. Nocedal (1999), Numerical optimization, Springer Science, 35(67-68), 7.

Zupanski, M. (2005), Maximum likelihood ensemble filter: Theoretical aspects, Monthly Weather Review, 133(6), 1710-1726.

Zupanski, M., I. M. Navon, and D. Zupanski (2008), The Maximum Likelihood Ensemble Filter as a non-differentiable minimization algorithm, Quarterly Journal of the Royal Meteorological Society, 134(633), 1039-1050. 